\title{
Synthesis and Fluorescent Properties of a New Class of Heterocycles of Isoindole Fused Imidazoles with Phenolic Subunits
}

\author{
Suven Das, Roland Fröhlich and Animesh Pramanik * \\ Department of Chemistry, University of Calcutta, 92, A. P. C. Road, Kolkata-700 009, India. \\ Organisch-Chemisches Institut, Universität Münster, Corrensstraße 40, D-48149 Münster, \\ Germany
}

\section{Supporting Information}

\section{Table of Contents}

\section{Contents page}

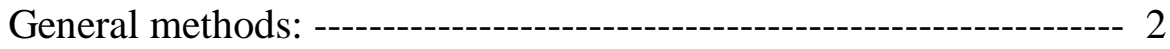

Procedure for the preparation of $\mathbf{4 a - m : ~ - - - - - - - - - - - - - - - - - - - - - - - - - - - - - - - - ~} 2$

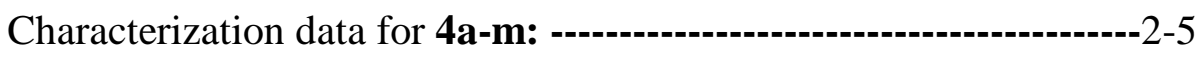

Procedure for the preparation of $\mathbf{4 n}$ and 4o: ------------------------------5

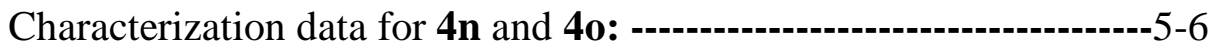

Copies of ${ }^{1} \mathrm{H}$ and ${ }^{13} \mathrm{C}$ NMR spectra of 4a-o: ------------------------ 7-36

Fluorescent emission and excitation spectra of 4a-o: ----------------- 37-51

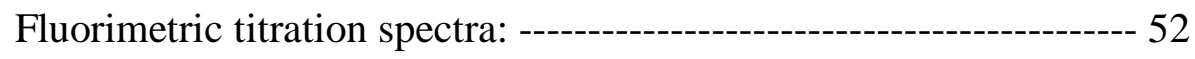

X-ray data for $\mathbf{4 f}$ and enlarged image of stained cells: ----------------- 53 
Melting points were determined in open capillary tubes and are uncorrected. All of the reagents and solvents are commercially available and were used as purchased, without further purification. Reaction products were purified by column chromatography, using silica gel (60-120 mesh) as stationary phase, eluting with petroleum ether/ethylacetate mixures. ${ }^{1} \mathrm{H}$ NMR and ${ }^{13} \mathrm{C}$ NMR were recorded with a Bruker Avance $300(300 \mathrm{MHz})$ or Bruker DRX-500 (500 MHz) spectrometers in the solvents indicated. Splitting patterns are designated as s (singlet), d (doublet), t (triplet), q (quartet), m (multiplet), bs (broad singlet) or dd (double doublet). Elemental analyses were performed on Perkin Elmer 240C analyzer. IR spectra were examined in Perkin Elmer-782 spectrophotometer. UV/Vis spectra were recorded with a Hitachi U-3501 Spectrophotometer. All the fluorescence measurements were made with a Perkin Elmer LS50B Luminescence Spectrometer. Absorption and emission spectra were recorded at $298 \mathrm{~K}$ at a concentration $10^{-6} \mathrm{M}$ for fluorescence quantum yields which were calculated according to the following equation $^{1}$

$\Phi_{\text {sample }}=\Phi_{\text {std }}\left[\mathrm{I}_{\text {sample }} / \mathrm{I}_{\text {std }}\right]\left[\mathrm{A}_{\text {std }} / \mathrm{A}_{\text {sample }}\right]\left[\eta_{\text {sample }} / \eta_{\text {std }}\right]^{2}$

In this equation $\Phi_{\text {sample }}$ and $\Phi_{\text {std }}$ are the quantum yields of sample and standard, respectively; $I_{\text {sample }}$ and $I_{s t d}$ are the integrated emission intensities of the sample and standard, respectively; $\mathrm{A}_{\text {std }}$ and $\mathrm{A}_{\text {sample }}$ are the absorbance of the sample and standard, respectively; and $\eta_{\text {sample }}$ and $\eta_{\text {std }}$ are the refractive indexes of the sample and standard solution, respectively.

\section{Typical procedure for the preparation of 1-aryl-3,5-dioxo-1H-imidazo-[3,4-}

b]isoindole, 4a-m: A mixture of ninhydrin (0.25 g, $1.4 \mathrm{mmol})$ and phenol, $1 \mathrm{a}$ (4.2 mmol) was refluxed in $\mathrm{AcOH}(6.0 \mathrm{ml})$ till the formation of adduct $\mathbf{2 a}$ was complete (monitored by TLC $)^{2}$. Then urea $(1.0 \mathrm{~g}, 16.6 \mathrm{mmol})$ was added to the above reaction mixture which was refluxed further for $2.5 \mathrm{~h}$. The reaction mixture turned into red color. The cold reaction mixture was poured into ice cold water. The yellow solid product was filtered and purified by column chromatography over silica gel (petroleum ether/ethylacetate $70 / 30 \mathrm{v} / \mathrm{v}$ ) to give $0.31 \mathrm{~g}$ of $\mathbf{4 a}$ ( $80 \%$ yield). The compound was crystallized from acetone. 
4a: yellow needles; mp: 305-307 ${ }^{0} \mathrm{C}$; IR (KBr): 3078, 1768, 1677, 1350, $736 \mathrm{~cm}^{-1}$; ${ }^{1} \mathrm{H}$ NMR (300 MHz, DMSO- $\left.d_{6}\right) \delta 10.64$ (bs, -NH), 7.80 ( d, $\left.J=7.8 \mathrm{~Hz}, 1 \mathrm{H}\right), 7.65$ (t, $J=7.6$ $\mathrm{Hz}, 1 \mathrm{H}), 7.47(\mathrm{~d}, J=7.6 \mathrm{~Hz}, 1 \mathrm{H}), 7.41-7.32(\mathrm{~m}, 3 \mathrm{H}), 7.05(\mathrm{~d}, J=8.2 \mathrm{~Hz}, 1 \mathrm{H}), 6.96(\mathrm{t}, J=$ $7.5 \mathrm{~Hz}, 1 \mathrm{H}) ;{ }^{13} \mathrm{C}$ NMR (75 MHz, DMSO-d $) \delta 157.4,152.3,144.5,131.3,129.1,128.3$, $127.8,126.6,124.2,122.4,119.0,117.6,116.4,113.8,113.4,111.8$; Anal calcd for $\mathrm{C}_{16} \mathrm{H}_{10} \mathrm{~N}_{2} \mathrm{O}_{3}, \mathrm{C}, 69.06 ; \mathrm{H}, 3.62 ; \mathrm{N}, 10.07$. Found C, 68.99; H, 3.58; N, 10.02.

4b: yellow crystals; mp: $254-256{ }^{0} \mathrm{C}$; IR (KBr): 3145, 1778, 1665, 1345, $743 \mathrm{~cm}^{-1}$; ${ }^{1} \mathrm{H}$ NMR (300 MHz, DMSO- $\left.d_{6}\right) \delta 10.75$ (bs, -NH), 9.13 (bs, -OH), 7.82 (d, $\left.J=7.7 \mathrm{~Hz}, 1 \mathrm{H}\right)$, $7.65(\mathrm{t}, J=7.6 \mathrm{~Hz}, 1 \mathrm{H}), 7.40(\mathrm{t}, J=7.6 \mathrm{~Hz}, 1 \mathrm{H}), 7.34-7.25(\mathrm{~m}, 3 \mathrm{H}), 6.91(\mathrm{t}, J=7.6 \mathrm{~Hz}$, $1 \mathrm{H}), 2.28(3 \mathrm{H}, \mathrm{s}) ;{ }^{13} \mathrm{C} \mathrm{NMR}\left(75 \mathrm{MHz}, \mathrm{DMSO}-d_{6}\right) \delta 160.8,153.3,148.0,134.8,132.9$, 132.3, 131.1, 127.6 (2C), 126.6, 125.7, 122.1, 121.0, 120.2, 117.4, 116.0, 17.1; Anal calcd for $\mathrm{C}_{17} \mathrm{H}_{12} \mathrm{~N}_{2} \mathrm{O}_{3}, \mathrm{C}, 69.86 ; \mathrm{H}, 4.14 ; \mathrm{N}, 9.58$. Found C, 69.78; H, 4.07; N, 9.53.

4c: yellow crystals; mp: 289-291 ${ }^{0} \mathrm{C}$; IR (KBr): 3229, 1751, 1667, 1348, $746 \mathrm{~cm}^{-1}$; ${ }^{1} \mathrm{H}$ NMR (300 MHz, DMSO-d $\left.d_{6}\right) 10.45$ (bs, -NH), $7.81(\mathrm{~d}, J=7.9 \mathrm{~Hz}, 1 \mathrm{H}), 7.66$ (t, $J=7.7$ $\mathrm{Hz}, 1 \mathrm{H}), 7.42-7.38$ (m, 2H), 7.27 (s, 1H), 7.15 (d, J=7.9 Hz, 1H), 6.95 (d, J = 8.3 Hz, 1H), $2.08(\mathrm{~s}, 3 \mathrm{H}) ;{ }^{13} \mathrm{C}$ NMR (75 MHz, DMSO- $\left.d_{6}\right) \delta 160.8,153.3,147.8,134.7,132.5$, 132.2, 131.1, 130.0, 128.4, 127.6, 125.7, 122.5, 121.1, 117.1, 116.7, 114.8, 20.4; Anal calcd for $\mathrm{C}_{17} \mathrm{H}_{12} \mathrm{~N}_{2} \mathrm{O}_{3}, \mathrm{C}, 69.86 ; \mathrm{H}, 4.14 ; \mathrm{N}, 9.58$. Found C, 69.80; H, 4.05; N, 9.50.

4d: yellow needles; mp: 221-222 ${ }^{0} \mathrm{C}$; IR (KBr): 3246, 1758, 1670, 1346, $746 \mathrm{~cm}^{-1}$; ${ }^{1} \mathrm{H}$ NMR (300 MHz, $\left.\mathrm{CDCl}_{3}+\mathrm{DMSO}_{6}\right) \delta 7.85(\mathrm{~d}, J=7.6 \mathrm{~Hz}, 1 \mathrm{H}), 7.59-7.49(\mathrm{~m}, 2 \mathrm{H}), 7.36$ $(\mathrm{d}, J=7.3 \mathrm{~Hz}, 1 \mathrm{H}), 7.07(\mathrm{dd}, J=8.3,2.0 \mathrm{~Hz}, 1 \mathrm{H}), 7.03(\mathrm{~s}, 1 \mathrm{H}), 6.95(\mathrm{~d}, J=8.3 \mathrm{~Hz}, 1 \mathrm{H})$, $2.16(\mathrm{~s}, 3 \mathrm{H}) ;{ }^{13} \mathrm{C}$ NMR $\left(75 \mathrm{MHz}, \mathrm{CDCl}_{3}+\mathrm{DMSO}-d_{6}\right) \delta 160.3,152.3,147.1,133.3$, $131.8,131.1,130.4,128.5,128.1,126.4,126.2$, 125.8, 124.9, 120.8, 116.0, 113.7, 19.7; Anal calcd for $\mathrm{C}_{17} \mathrm{H}_{12} \mathrm{~N}_{2} \mathrm{O}_{3}, \mathrm{C}, 69.86 ; \mathrm{H}, 4.14 ; \mathrm{N}, 9.58$. Found C, 69.79, H, 4.10; N, 9.52. 4e: yellow crystals; mp: 276-277 ${ }^{0} \mathrm{C}$; IR (KBr): 3263, 1759, 1670, 1349, $747 \mathrm{~cm}^{-1}$; $1 \mathrm{H}$ NMR ( $300 \mathrm{MHz}$, Acetone- $\left.d_{6}\right) \delta 9.65$ (bs, -NH), 8.64 (bs, -OH), 7.85 (d, J=7.7 Hz, 1H), 7.67-7.61 (m, 2H), 7.48-7.42 (m, 1H), $7.26(\mathrm{dd}, J=7.8,1.5 \mathrm{~Hz}, 1 \mathrm{H}), 7.15(\mathrm{dd}, J=8.1,1.4$ $\mathrm{Hz}, 1 \mathrm{H}), 7.03(\mathrm{t}, J=7.9 \mathrm{~Hz}, 1 \mathrm{H}), 3.95(\mathrm{~s}, 3 \mathrm{H}) ;{ }^{13} \mathrm{C}$ NMR $\left(75 \mathrm{MHz}\right.$, Acetone- $\left.d_{6}\right) \delta 160.5$, $148.5,147.6,145.0,134.1,132.5,131.3,126.2$, 125.2, 122.3 , 121.0, 120.6, 119.6, 117.3, 
115.2, 112.9, 55.9; Anal calcd for $\mathrm{C}_{17} \mathrm{H}_{12} \mathrm{~N}_{2} \mathrm{O}_{4}, \mathrm{C}, 66.23 ; \mathrm{H}, 3.92 ; \mathrm{N}, 9.09$. Found $\mathrm{C}$, $66.17, \mathrm{H}, 3.86 ; \mathrm{N}, 9.02$.

4f: yellow crystals; mp: $298-300{ }^{0} \mathrm{C}$; IR (KBr): $3355,1756,1761,1343,766 \mathrm{~cm}^{-1} ;{ }^{1} \mathrm{H}$ NMR (300 MHz, DMSO- $\left.d_{6}\right) \delta 10.39$ (bs, -NH), $7.82(\mathrm{~d}, J=7.6 \mathrm{~Hz}, 1 \mathrm{H}), 7.68(\mathrm{t}, J=7.3$ $\mathrm{Hz}, 1 \mathrm{H}), 7.45-7.39$ (m, 2H), 7.02-6.93 (m, 3H), 3.69 (s, 3H), ${ }^{13} \mathrm{C}$ NMR (75 MHz, DMSO$\left.d_{6}\right) \delta 160.8,152.5,149.5,147.9,134.6,132.5,131.1,127.6,125.7,122.8,120.8,118.1$, 117.8, 117.4, 115.3, 113.9, 56.0; Anal calcd for $\mathrm{C}_{17} \mathrm{H}_{12} \mathrm{~N}_{2} \mathrm{O}_{4}, \mathrm{C}, 66.23 ; \mathrm{H}, 3.92 ; \mathrm{N}, 9.09$. Found $\mathrm{C}, 66.18 ; \mathrm{H}, 3.85 ; \mathrm{N}, 9.03$.

4g: yellow needles; mp: $274-275{ }^{0} \mathrm{C}$; IR (KBr): 3206, 1767, 1679,1343, $740 \mathrm{~cm}^{-1} ;{ }^{1} \mathrm{H}$ NMR (300 MHz, DMSO-d6) $\delta 10.50$ (bs, -NH), 7.82 (d, $J=7.6 \mathrm{~Hz}, 1 \mathrm{H}), 7.66$ (t, $J=7.4$ $\mathrm{Hz}, 1 \mathrm{H}), 7.54(\mathrm{dd}, J=7.9,1.0 \mathrm{~Hz}, 1 \mathrm{H}), 7.45-7.39(\mathrm{~m}, 2 \mathrm{H}), 7.31(\mathrm{~d}, J=7.7 \mathrm{~Hz}, 1 \mathrm{H}), 7.02$ $(\mathrm{t}, J=7.8 \mathrm{~Hz}, 1 \mathrm{H}) ;{ }^{13} \mathrm{C}$ NMR $\left(75 \mathrm{MHz}\right.$, DMSO- $\left.d_{6}\right) \delta 160.8,151.1,147.8,134.9,132.1$, $131.8,131.2,129.0,128.0,125.8,122.4,122.2,121.2,119.7,118.2,118.1$; Anal calcd for $\mathrm{C}_{16} \mathrm{H}_{9} \mathrm{ClN}_{2} \mathrm{O}_{3}, \mathrm{C}, 61.45 ; \mathrm{H}, 2.90 ; \mathrm{N}, 8.96$. Found C, 61.39; H, 2.86; N, 8.87.

4h: yellow needles; mp: $308-310{ }^{0} \mathrm{C}$; IR (KBr): $3430,1780,1689,1348,738 \mathrm{~cm}^{-1} ;{ }^{1} \mathrm{H}$ NMR (300 MHz, DMSO- $d_{6}$ ) d 10.87 (bs, -NH), 7.81 (d, $\left.J=7.6 \mathrm{~Hz}, 1 \mathrm{H}\right), 7.67$ (t, $J=7.6$ $\mathrm{Hz}, 1 \mathrm{H}), 7.49$ (d, $J=2.4 \mathrm{~Hz}, 1 \mathrm{H}), 7.44-7.35(\mathrm{~m}, 3 \mathrm{H}), 7.07$ (d, $J=8.8 \mathrm{~Hz}, 1 \mathrm{H}) ;{ }^{13} \mathrm{C} \mathrm{NMR}$ $\left(75 \mathrm{MHz}, \mathrm{DMSO}-d_{6}\right) \delta 160.8,154.5,147.7,134.7,132.3,131.2,131.1,129.2,127.9$, 125.8, 123.2, 122.8, 119.1, 118.4, 118.0, 116.9; Anal calcd for $\mathrm{C}_{16} \mathrm{H}_{9} \mathrm{ClN}_{2} \mathrm{O}_{3}, \mathrm{C}, 61.45$; H, 2.90; N, 8.96. Found C, 61.39; H, 2.85; N, 8.91.

4i: yellow needles; mp: $316-318{ }^{0} \mathrm{C}$; IR (KBr): $3319,1773,1689,1341,735 \mathrm{~cm}^{-1}$; ${ }^{1} \mathrm{H}$ NMR (300 MHz, DMSO- $\left.d_{6}\right) \delta 10.77$ (bs, -NH), $7.82(\mathrm{~d}, J=7.5 \mathrm{~Hz}, 1 \mathrm{H}), 7.68(\mathrm{t}, J=7.4$ $\mathrm{Hz}, 1 \mathrm{H}), 7.47$ (s, 1H), 7.43-7.36 (m, 2H), 7.00 (s, 1H), 2.32 (s, 3H); ${ }^{13} \mathrm{C}$ NMR (75 MHz, DMSO- $\left.d_{6}\right) \delta 160.7,154.3,147.7,138.8,134.6,132.3,131.2,129.4,127.7,125.7,123.7$, 122.7, 119.3, 119.1, 117.6, 114.6, 20.3; Anal calcd for $\mathrm{C}_{17} \mathrm{H}_{11} \mathrm{ClN}_{2} \mathrm{O}_{3}, \mathrm{C}, 62.49 ; \mathrm{H}, 3.39$; N, 8.57. Found C, 62.42; H, 3.33; N, 8.51.

4j: yellow needles; mp: $306-307{ }^{0} \mathrm{C}$; IR (KBr): $3268,1779,1687,1347,740 \mathrm{~cm}^{-1}$; ${ }^{1} \mathrm{H}$ NMR (300 MHz, DMSO- $\left.d_{6}\right) \delta 10.87$ (bs, -NH), $7.83(\mathrm{~d}, J=7.5 \mathrm{~Hz}, 1 \mathrm{H}), 7.69(\mathrm{t}, J=7.4$ $\mathrm{Hz}, 1 \mathrm{H}), 7.61$ (s, 1H), 7.51 (d, $J=8.7 \mathrm{~Hz}, 1 \mathrm{H}), 7.44$ (t, $J=7.5 \mathrm{~Hz}, 1 \mathrm{H}), 7.38$ (d, $J=7.8$ $\mathrm{Hz}, 1 \mathrm{H}), 7.02(\mathrm{~d}, J=8.7 \mathrm{~Hz}, 1 \mathrm{H}) ;{ }^{13} \mathrm{C}$ NMR $\left(75 \mathrm{MHz}\right.$, DMSO- $\left.d_{6}\right) \delta 160.8,154.9,147.7$, 134.7, 134.0, 132.3, 132.0, 131.2, 127.9, 125.8, 122.8, 119.1, 118.9, 118.0, 117.5, 110.6; 
Anal calcd for $\mathrm{C}_{16} \mathrm{H}_{9} \mathrm{BrN}_{2} \mathrm{O}_{3}, \mathrm{C}, 53.81 ; \mathrm{H}, 2.54 ; \mathrm{N}, 7.84$. Found C, 53.75; H, 2.48; N, 7.78 .

4k: yellow crystals; mp: 320-322 ${ }^{0} \mathrm{C}$; IR (KBr): 3311, 1683, 1357, $740 \mathrm{~cm}^{-1}$; ${ }^{1} \mathrm{H}$ NMR (300 MHz, DMSO-d $) \delta 9.87$ (s, CHO), $7.83(\mathrm{~d}, J=7.6 \mathrm{~Hz}, 1 \mathrm{H}), 7.73$ (d, $J=1.2 \mathrm{~Hz}$, 1H), $7.66(\mathrm{t}, J=7.6 \mathrm{~Hz}, 1 \mathrm{H}), 7.54(\mathrm{~d}, J=1.2 \mathrm{~Hz}, 1 \mathrm{H}), 7.42(\mathrm{t}, J=7.5 \mathrm{~Hz}, 1 \mathrm{H}), 7.36$ (d, $J$ $=7.8 \mathrm{~Hz}, 1 \mathrm{H}), 3.98(\mathrm{~s}, 3 \mathrm{H}) ;{ }^{13} \mathrm{C}$ NMR $\left(75 \mathrm{MHz}, \mathrm{DMSO}-d_{6}\right) \delta 191.0,160.4,150.6,148.7$, $147.4,134.5,131.8,130.9,128.3,127.6,126.1,125.4,122.4,118.9,117.7,115.2,110.8$, 56.4; Anal calcd for $\mathrm{C}_{18} \mathrm{H}_{12} \mathrm{~N}_{2} \mathrm{O}_{5}, \mathrm{C}, 64.29 ; \mathrm{H}, 3.60 ; \mathrm{N}, 8.33$. Found $\mathrm{C}, 64.23 ; \mathrm{H}, 3.53, \mathrm{~N}$, 8.28 .

4l: yellow crystals; mp: $314-316{ }^{0} \mathrm{C}$; IR (KBr): 3294, 1782, 1718, 1690, 1351, 1286, 737 $\mathrm{cm}^{-1} ;{ }^{1} \mathrm{H}$ NMR $\left(300 \mathrm{MHz}, \mathrm{DMSO}-d_{6}\right) \delta 8.07(\mathrm{~s}, 1 \mathrm{H}), 7.91(\mathrm{~d}, J=8.6 \mathrm{~Hz}, 1 \mathrm{H}), 7.81(\mathrm{~d}, J$ $=7.5 \mathrm{~Hz}, 1 \mathrm{H}), 7.65(\mathrm{t}, J=7.5 \mathrm{~Hz}, 1 \mathrm{H}), 7.40(\mathrm{t}, J=7.5 \mathrm{~Hz}, 1 \mathrm{H}), 7.34(\mathrm{~d}, J=7.7 \mathrm{~Hz}, 1 \mathrm{H})$, $7.12(\mathrm{~d}, J=8.6 \mathrm{~Hz}, 1 \mathrm{H}), 3.82(\mathrm{~s}, 3 \mathrm{H}) ;{ }^{13} \mathrm{C}$ NMR (75 MHz, DMSO- $\left.d_{6}\right) \delta 166.0,160.8$, $159.9,147.8,134.7,132.8,132.2,131.7,131.2,127.9,125.8,122.3,121.0,119.7,117.8$, 116.9, 115.4, 52.4; Anal calcd for $\mathrm{C}_{18} \mathrm{H}_{12} \mathrm{~N}_{2} \mathrm{O}_{5}, \mathrm{C}, 64.29 ; \mathrm{H}, 3.60 ; \mathrm{N}, 8.33$. Found $\mathrm{C}$, $64.22 ; \mathrm{H}, 3.55 ; \mathrm{N}, 8.25$.

4m: yellow needles; mp: 320-321 ${ }^{0} \mathrm{C}$; IR (KBr): 3323, 1771, 1707, 1349, 1294, 1251, $735 \mathrm{~cm}^{-1} ;{ }^{1} \mathrm{H}$ NMR $\left(500 \mathrm{MHz}\right.$, Acetone- $\left.d_{6}\right) \delta 8.34(\mathrm{~d}, J=2.1 \mathrm{~Hz}, 1 \mathrm{H}), 8.00(\mathrm{dd}, J=8.5$, $2.1 \mathrm{~Hz}, 1 \mathrm{H}), 7.85(\mathrm{~d}, J=7.6 \mathrm{~Hz}, 1 \mathrm{H}), 7.68(\mathrm{t}, J=8.0 \mathrm{~Hz}, 1 \mathrm{H}), 7.64(\mathrm{~d}, J=7.8 \mathrm{~Hz}, 1 \mathrm{H})$, $7.47(\mathrm{t}, J=8.0 \mathrm{~Hz}, 1 \mathrm{H}), 7.22(\mathrm{~d}, J=8.5 \mathrm{~Hz}, 1 \mathrm{H}), 4.35(\mathrm{q}, J=7.1 \mathrm{~Hz}, 2 \mathrm{H}), 1.36(\mathrm{t}, J=7.1$ $\mathrm{Hz}, 3 \mathrm{H}) ;{ }^{13} \mathrm{C}$ NMR $\left(125 \mathrm{MHz}, \mathrm{DMSO}-d_{6}\right) \delta 165.9,161.1,160.2,148.2,135.2,133.2$, $132.6,132.1,131.7,128.3,126.3,122.6,121.8,120.2,118.2,117.3,115.8,61.4,15.1$; Anal calcd for $\mathrm{C}_{19} \mathrm{H}_{14} \mathrm{~N}_{2} \mathrm{O}_{5}, \mathrm{C}, 65.14 ; \mathrm{H}, 4.03 ; \mathrm{N}, 8.00$. Found $\mathrm{C}, 65.08 ; \mathrm{H}, 3.95 ; \mathrm{N}, 7.92$.

Typical procedure for the preparation of $4 \mathbf{n}$ and 4o: Initially the adduct $2 \mathrm{n}$ and 20 were prepared and isolated following the reported procedure. ${ }^{3}$ Then a mixture of $\mathbf{2 n}$ $(0.50 \mathrm{~g}, 1.68 \mathrm{mmol})$ and urea $(1.0 \mathrm{~g}, 16.6 \mathrm{mmol})$ in $\mathrm{AcOH}(6.0 \mathrm{ml})$ was refluxed for $8 \mathrm{~h}$. The reaction mixture turned into red color. The cold reaction mixture was poured into ice cold water. The yellow solid product was filtered and purified by column chromatography over silica gel (petroleum ether/ethylacetate $70 / 30 \mathrm{v} / \mathrm{v}$ ) to give $0.34 \mathrm{~g}$ of 4n (62\% yield). The compound was crystallized from acetone. 
4n: yellow needles; mp: 312-314 ${ }^{0} \mathrm{C}$; IR (KBr): 3433, 1760, 1674, 1607, 1343, $744 \mathrm{~cm}^{-1}$; ${ }^{1} \mathrm{H}$ NMR (300 MHz, DMSO-d $) \delta 10.79(\mathrm{~s},-\mathrm{NH}), 7.77(\mathrm{~d}, J=7.4 \mathrm{~Hz}, 1 \mathrm{H}), 7.62$ (t, $J=$ $7.4 \mathrm{~Hz}, 1 \mathrm{H}), 7.44(\mathrm{~d}, J=8.3 \mathrm{~Hz}, 1 \mathrm{H}), 7.36(\mathrm{t}, J=7.5 \mathrm{~Hz}, 1 \mathrm{H}), 7.27(\mathrm{~d}, J=7.5 \mathrm{~Hz}, 1 \mathrm{H})$, 6.71-6.68 (m, 2H), $3.82\left(\mathrm{~s}, 6 \mathrm{H}, 2 \times \mathrm{CH}_{3}\right) ;{ }^{13} \mathrm{C}$ NMR (75 MHz, DMSO-d 6 ) $\delta 162.7,161.0$, 158.4 , 147.8, 134.8, 132.4, 131.0, 127.6, 125.8, 121.6, 120.7, 116.6, 109.1, 106.2, 99.2, 56.1, 56.0; Anal calcd for $\mathrm{C}_{18} \mathrm{H}_{14} \mathrm{~N}_{2} \mathrm{O}_{4}, \mathrm{C}, 67.07 ; \mathrm{H}, 4.38 ; \mathrm{N}, 8.69$. Found C, 67.02; H, $4.29 ; \mathrm{N}, 8.62$.

4o: yellow needles; mp: 286-287 ${ }^{0} \mathrm{C}$; IR (KBr): 3335, 1767, 1678, 1333, 1225, $746 \mathrm{~cm}^{-1}$; ${ }^{1} \mathrm{H}$ NMR (300 MHz, DMSO-d $)_{6} \delta 10.94(\mathrm{~s},-\mathrm{NH}), 7.83$ (d, $\left.J=7.6 \mathrm{~Hz}, 1 \mathrm{H}\right), 7.69$ (t, $J=$ $7.5 \mathrm{~Hz}, 1 \mathrm{H}), 7.43$ (t, $J=7.5 \mathrm{~Hz}, 1 \mathrm{H}), 7.33(\mathrm{~d}, J=7.7 \mathrm{~Hz}, 1 \mathrm{H}), 7.19-7.07$ (m, 3H), 3.81 (s, 3H), $3.78(\mathrm{~s}, 3 \mathrm{H}) ;{ }^{13} \mathrm{C}$ NMR (75 MHz, DMSO-d 6 ) $\delta 160.7,153.4,151.1,147.7,134.9$, $132.3,131.2,127.9,125.8,122.2,120.0,117.8,117.3,117.1,115.0,113.5,56.3,56.1$; Anal calcd for $\mathrm{C}_{18} \mathrm{H}_{14} \mathrm{~N}_{2} \mathrm{O}_{4}, \mathrm{C}, 67.07 ; \mathrm{H}, 4.38 ; \mathrm{N}, 8.69$. Found C, 67.00; H, 4.32; N, 8.65.

\section{Related references}

(1) Demas, J. N.; Crosbi, G. A. J. Phys. Chem. 1971, 75, 991.

(2) (a) Schmitt, G.; Dinh An, N.; Poupelin, J.-P.; Vebrel, J.; Laude, B. Synthesis 1984, 758. (b) Das, S.; Fröhlich, R.; Pramanik, A. Synlett 2006, 207.

(3) Kundu, S. K.; Pramanik, A.; Patra, A. Synlett 2002, 823. 


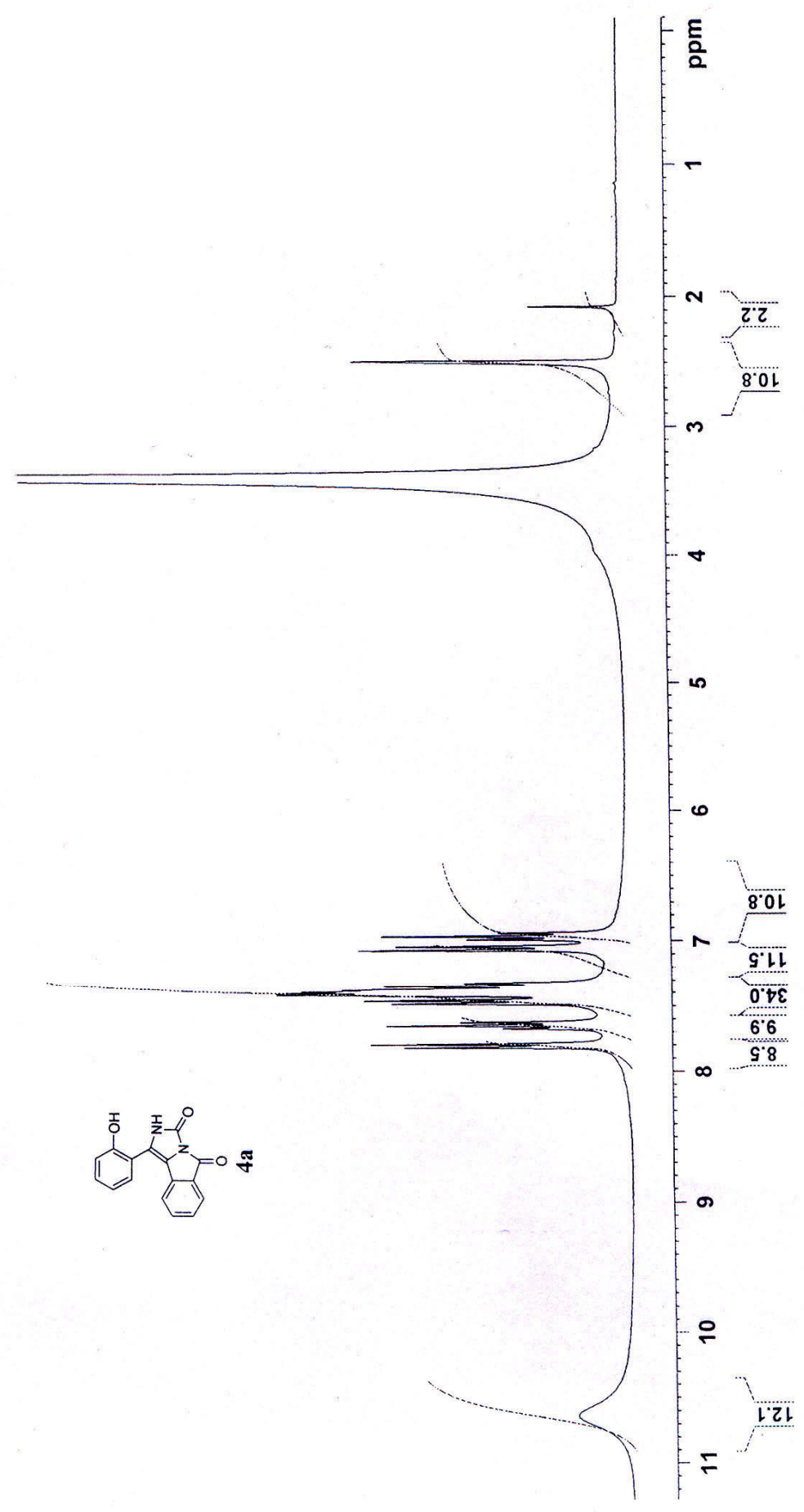



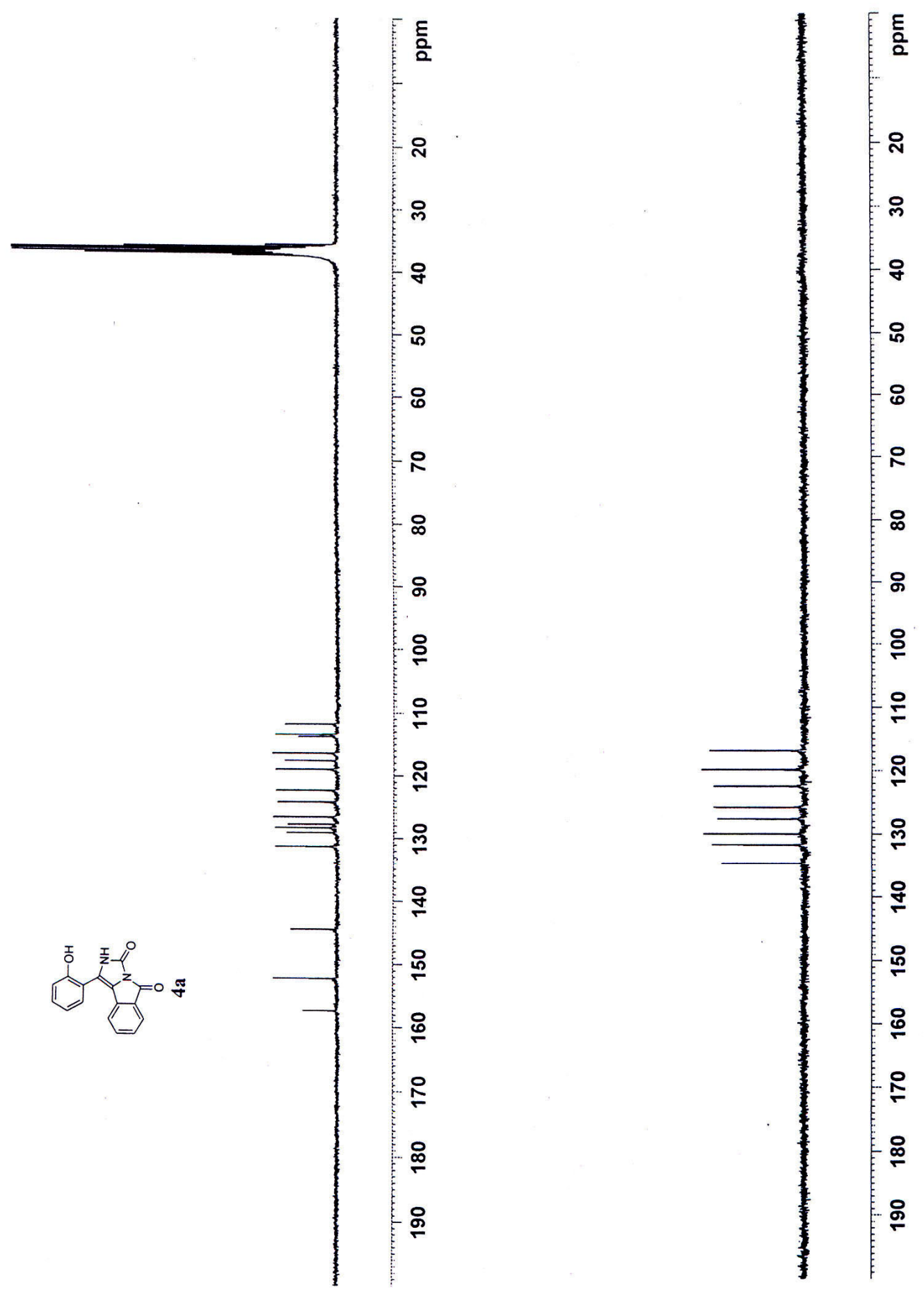


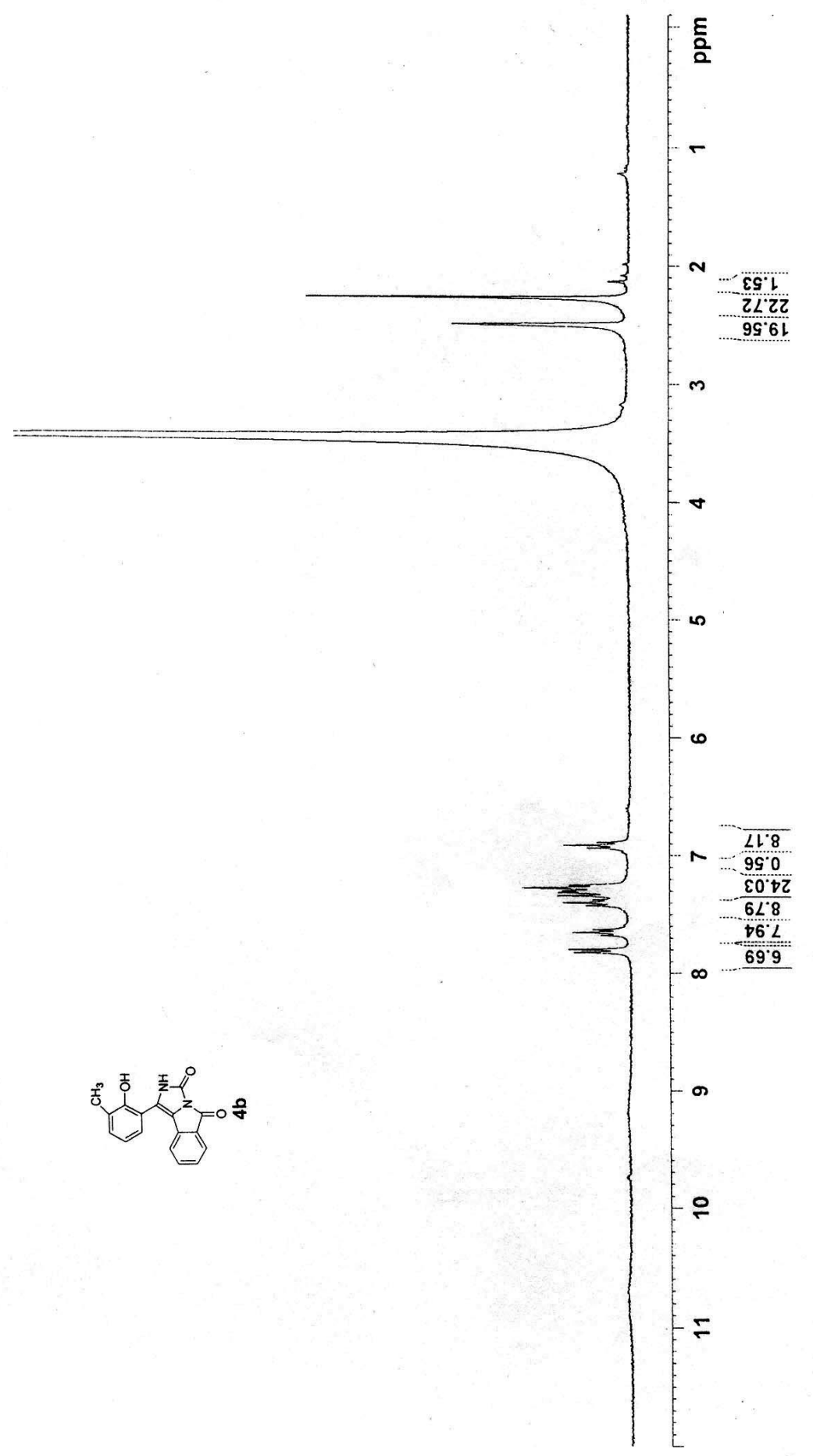



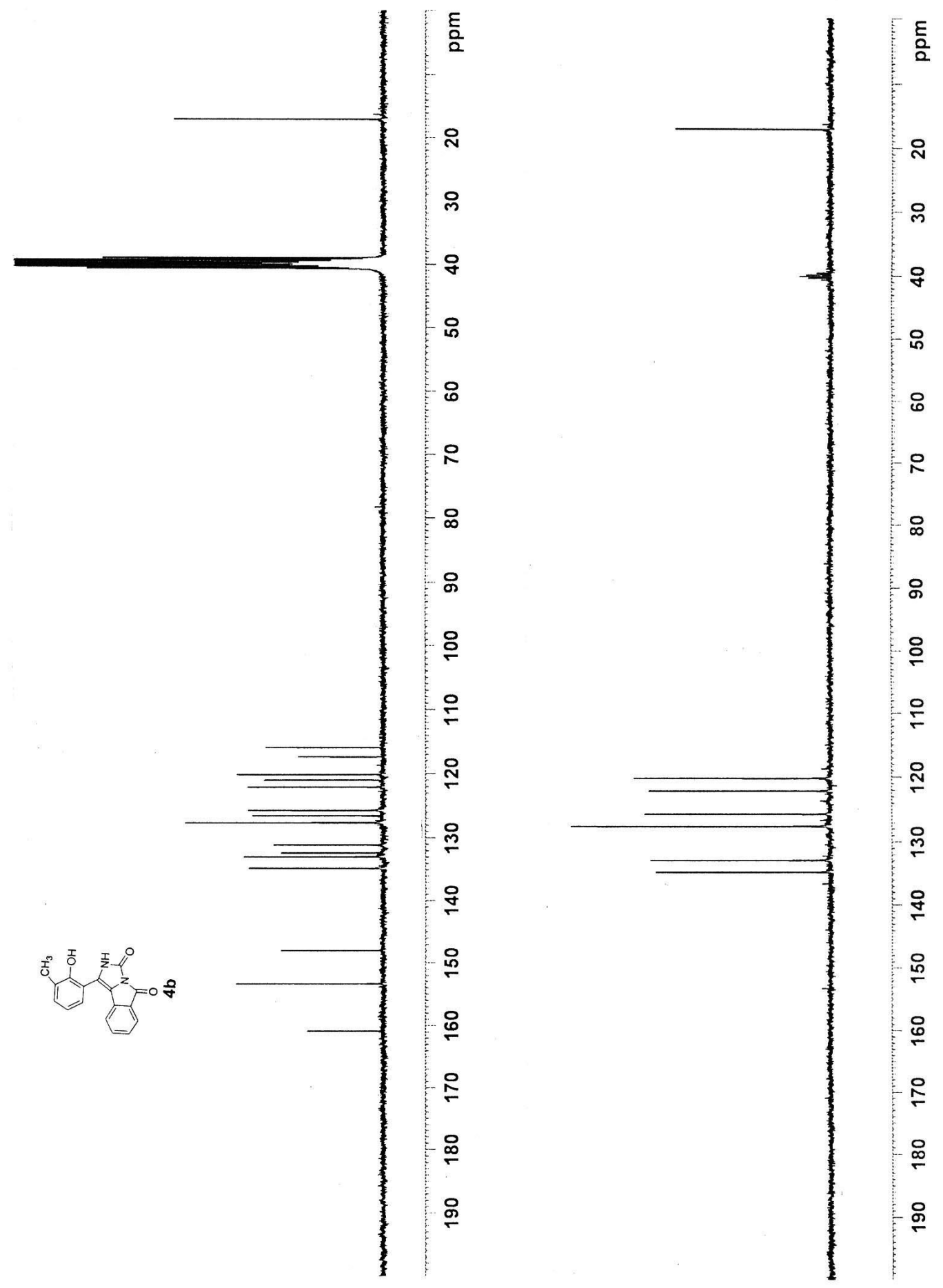


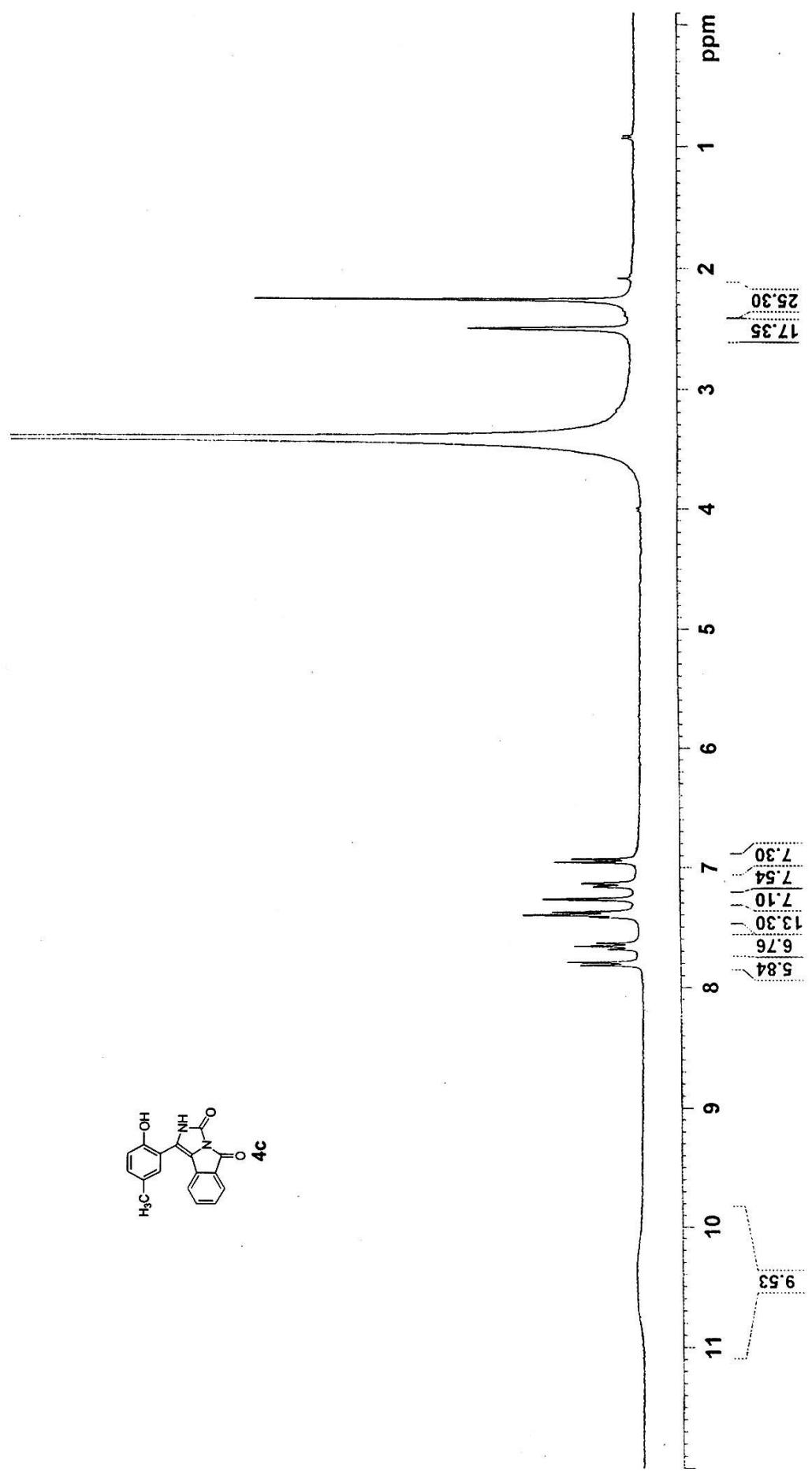



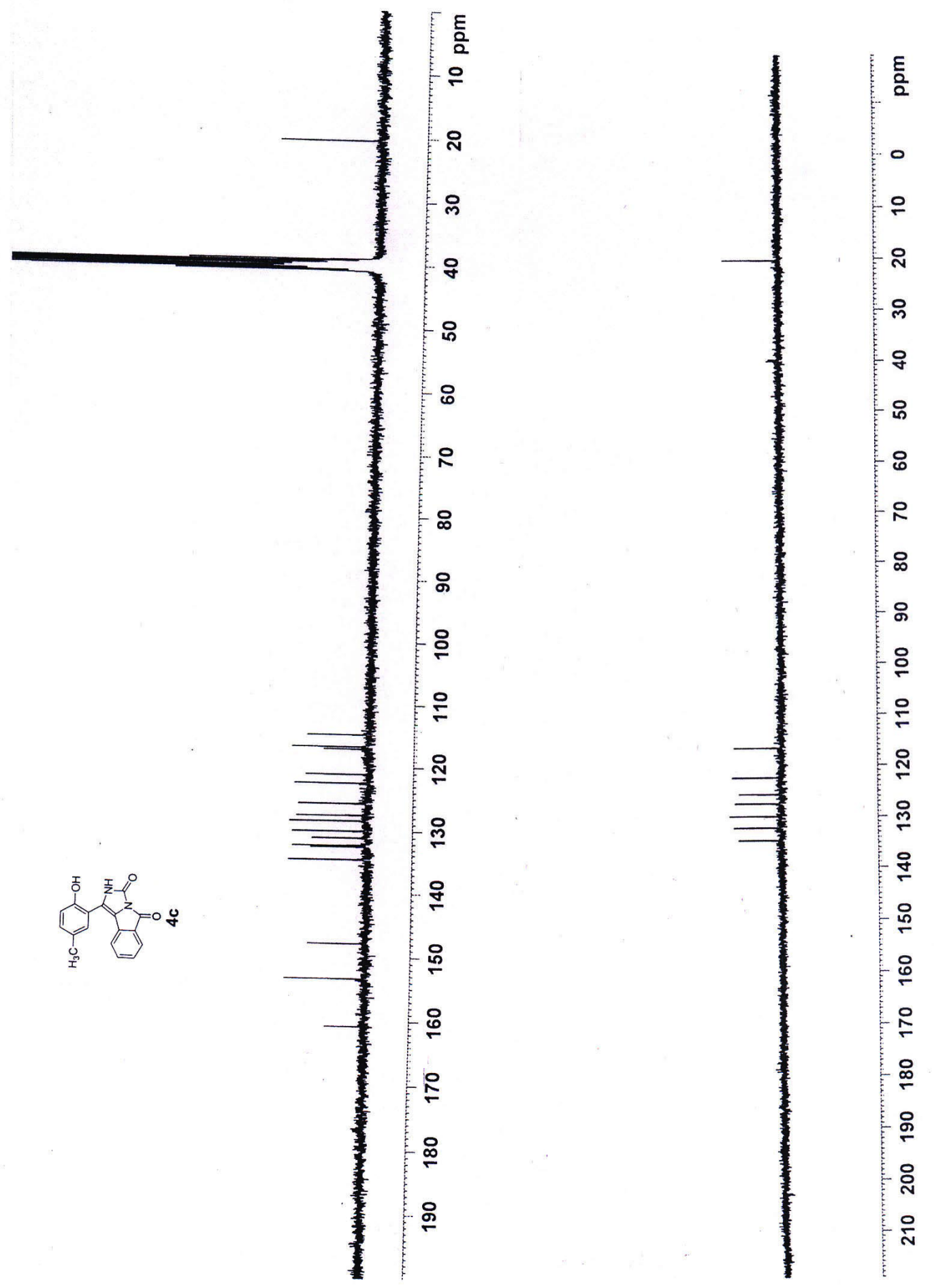


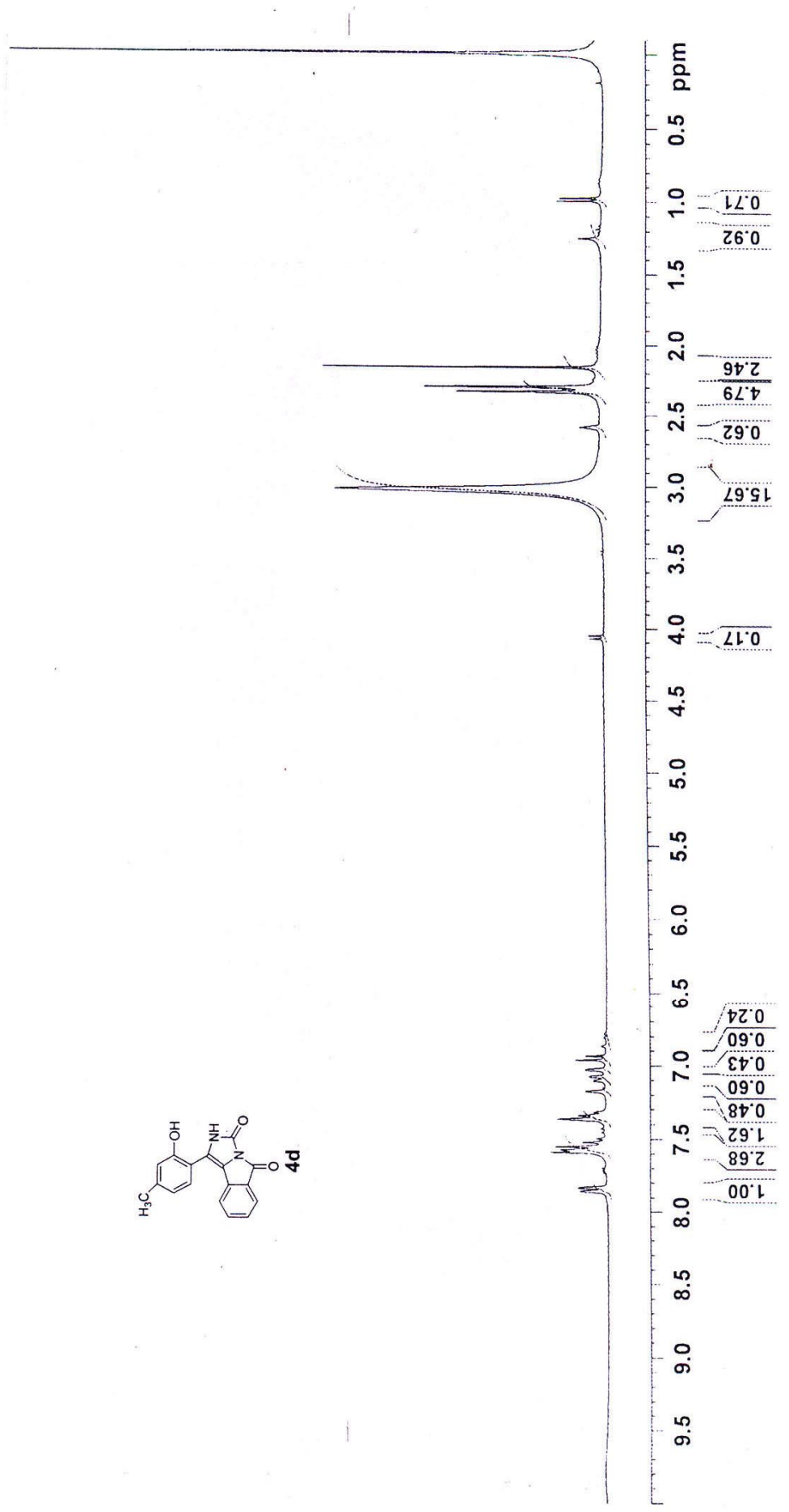



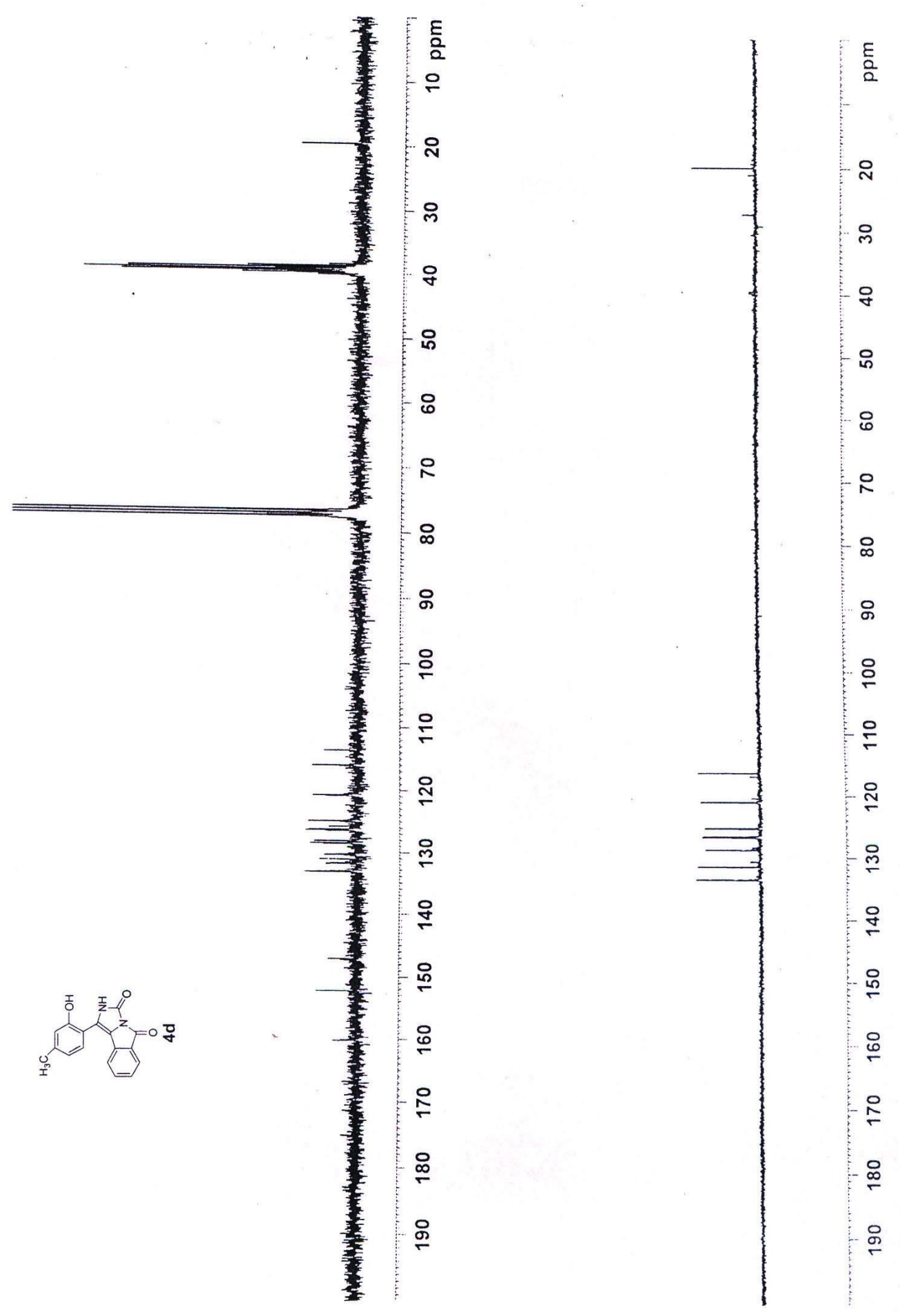


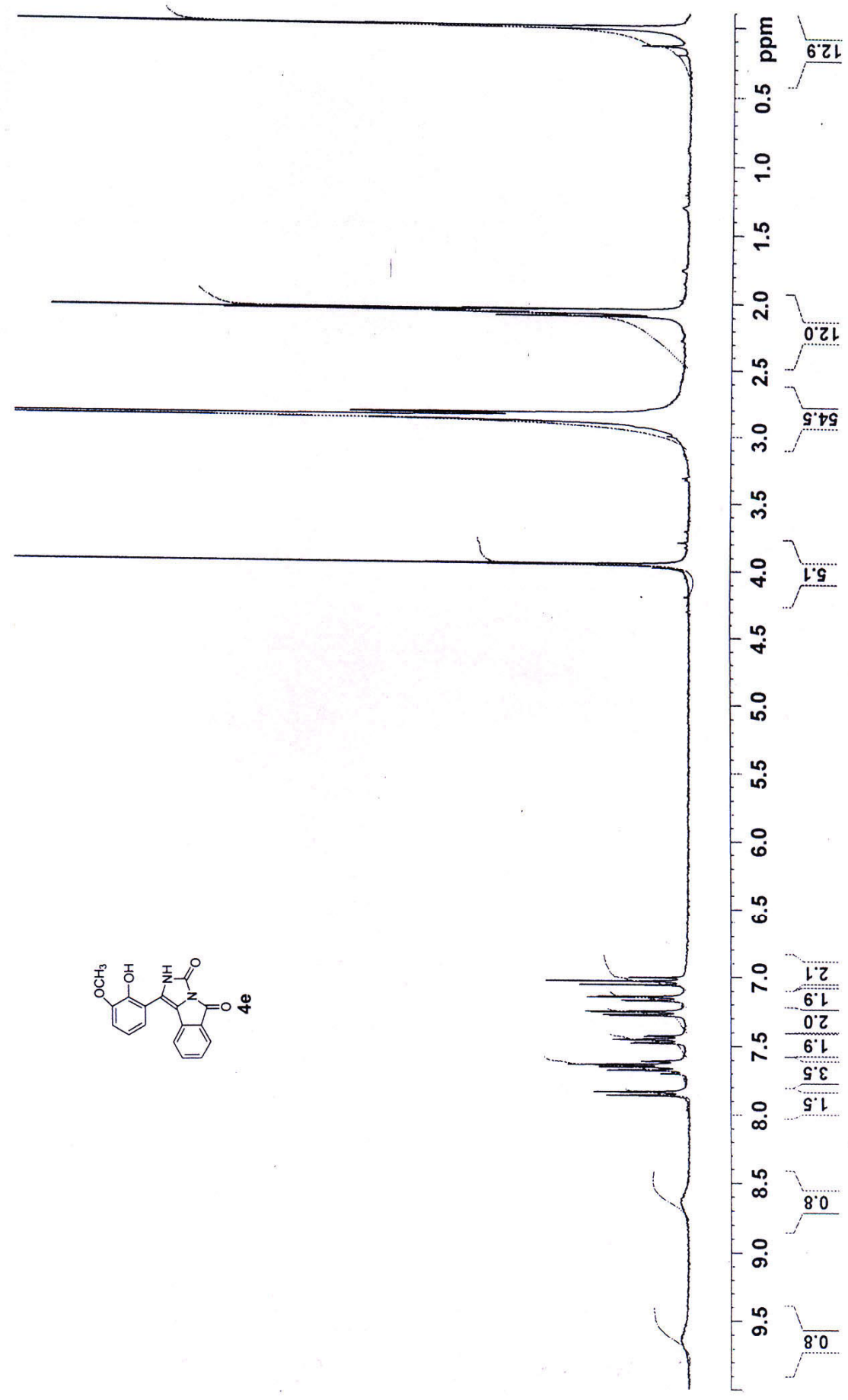



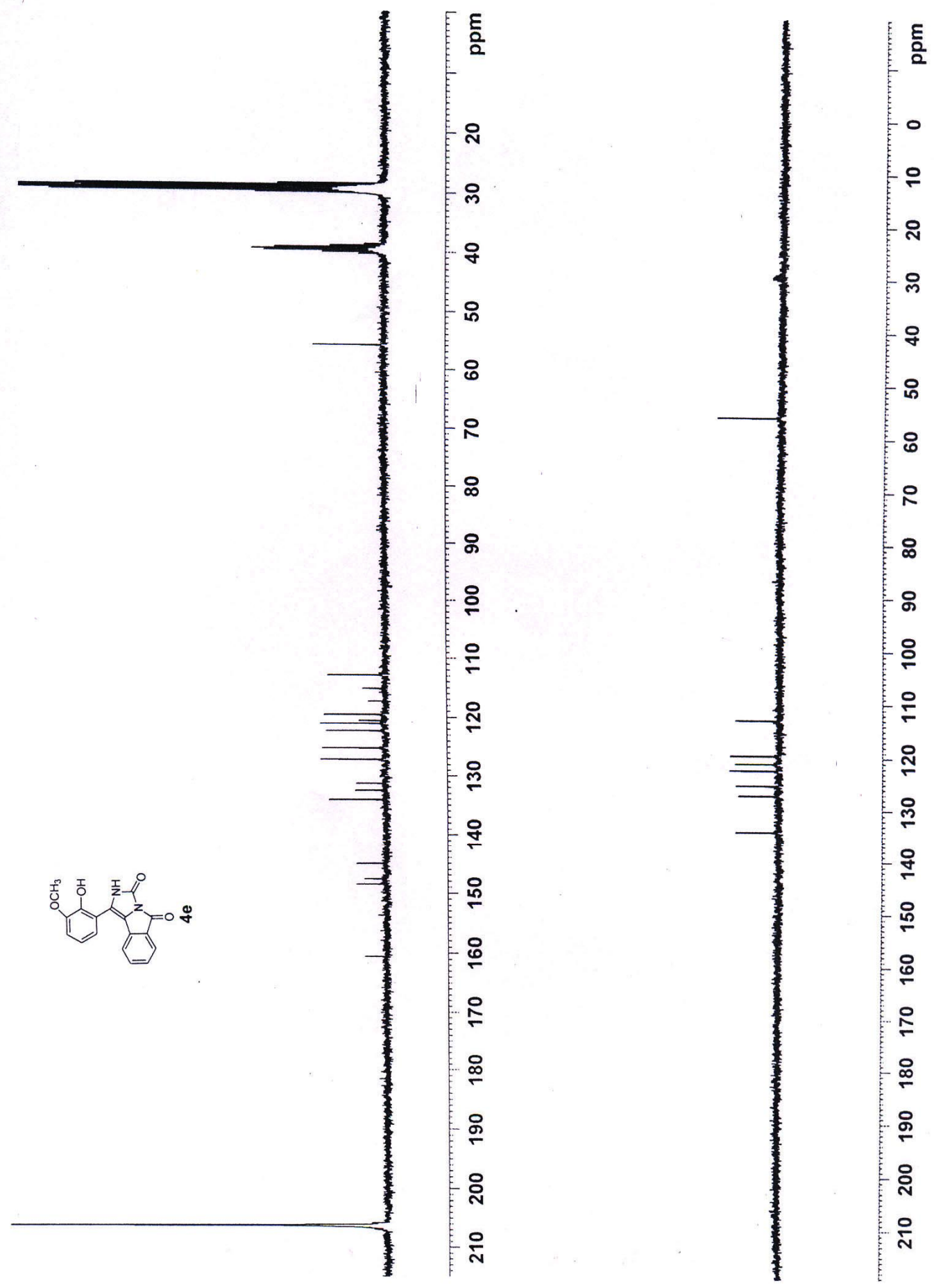


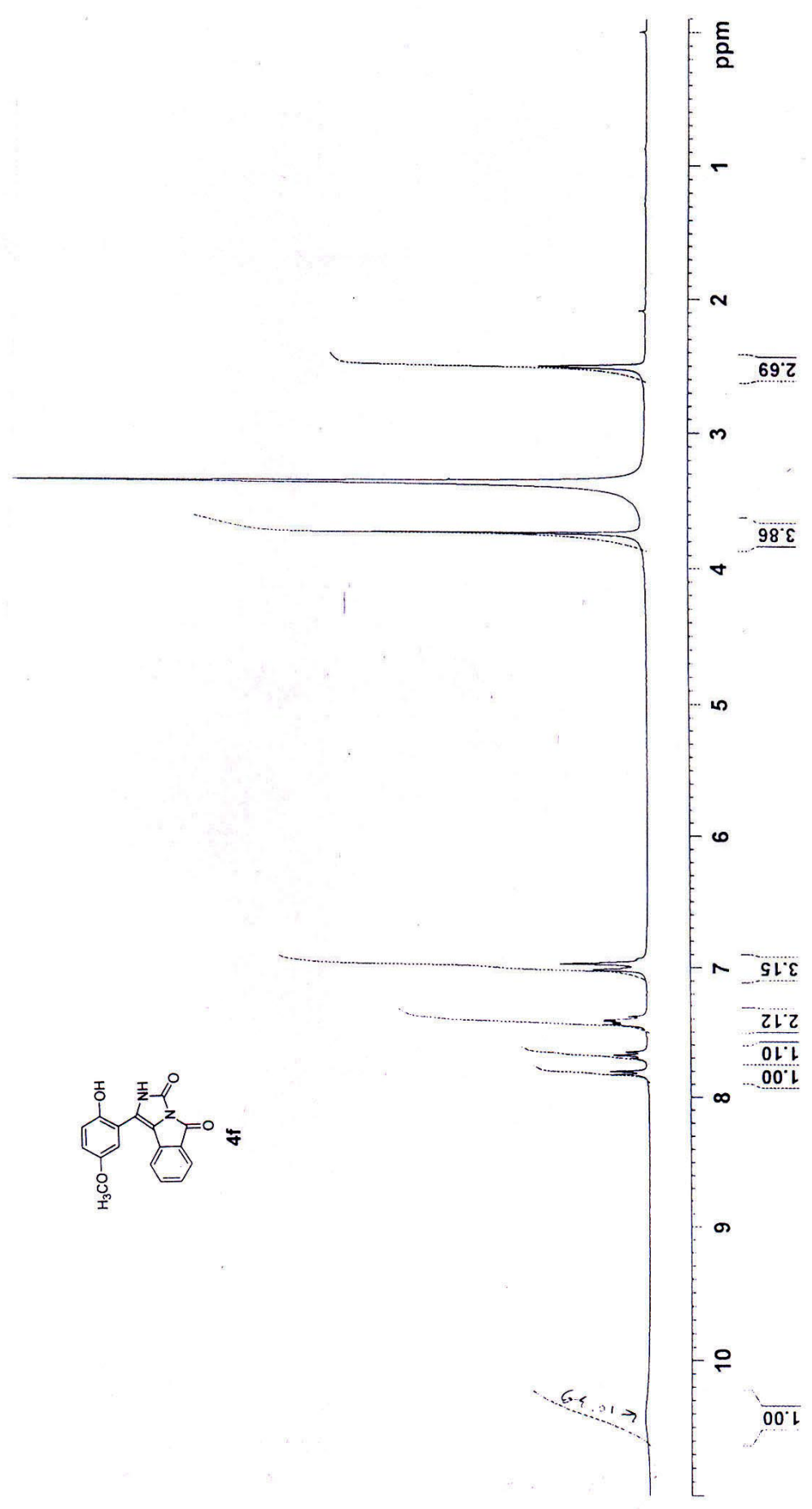



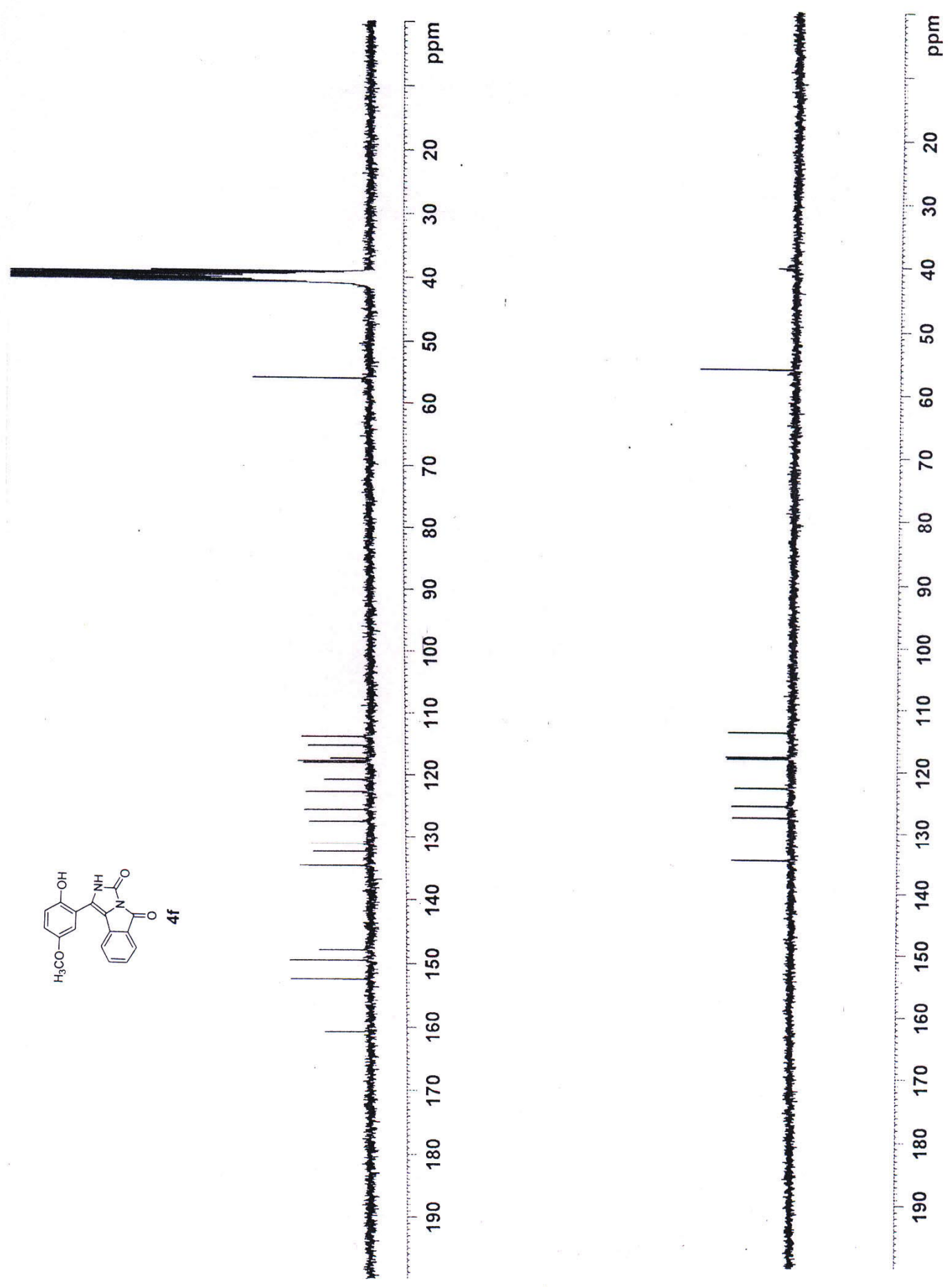


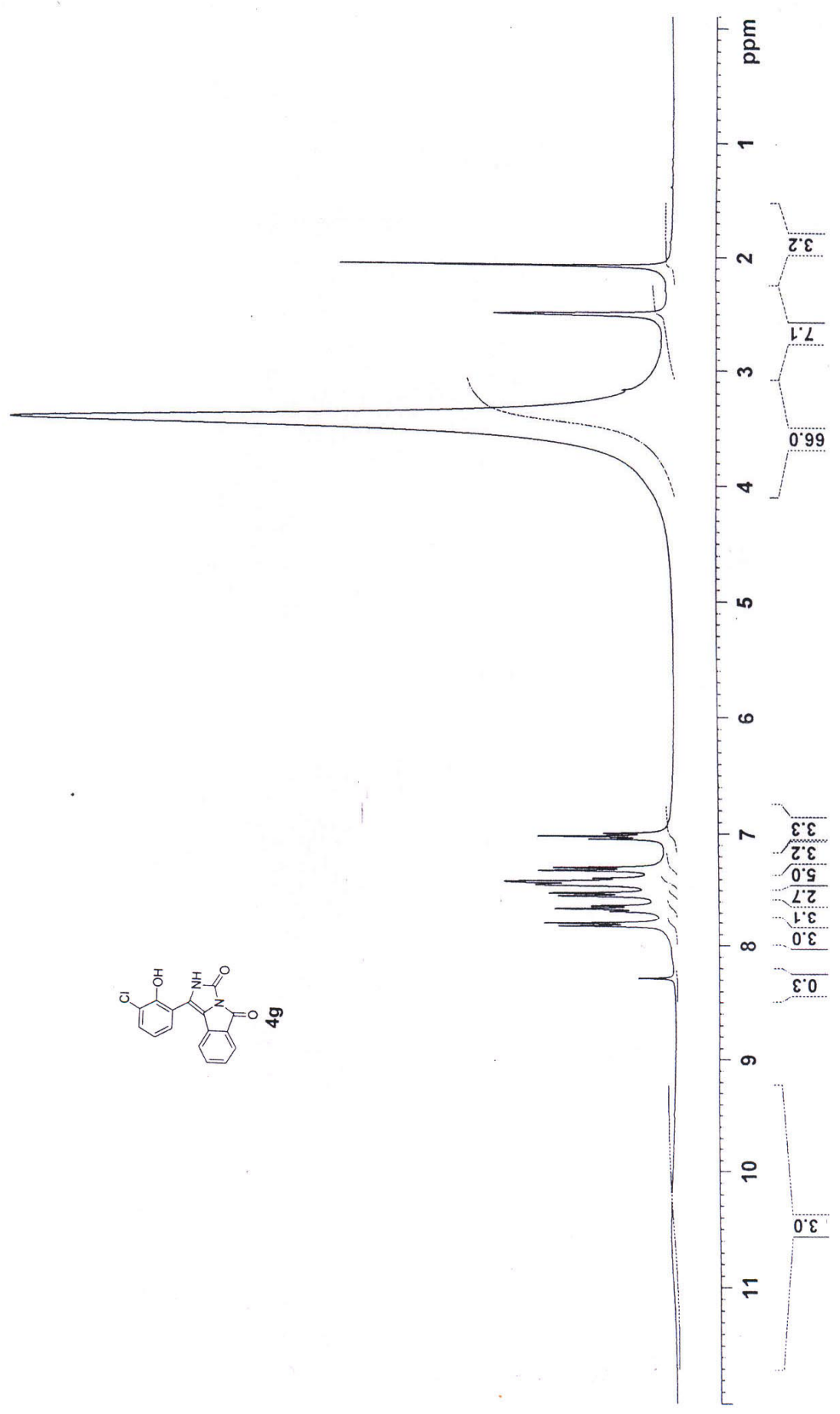




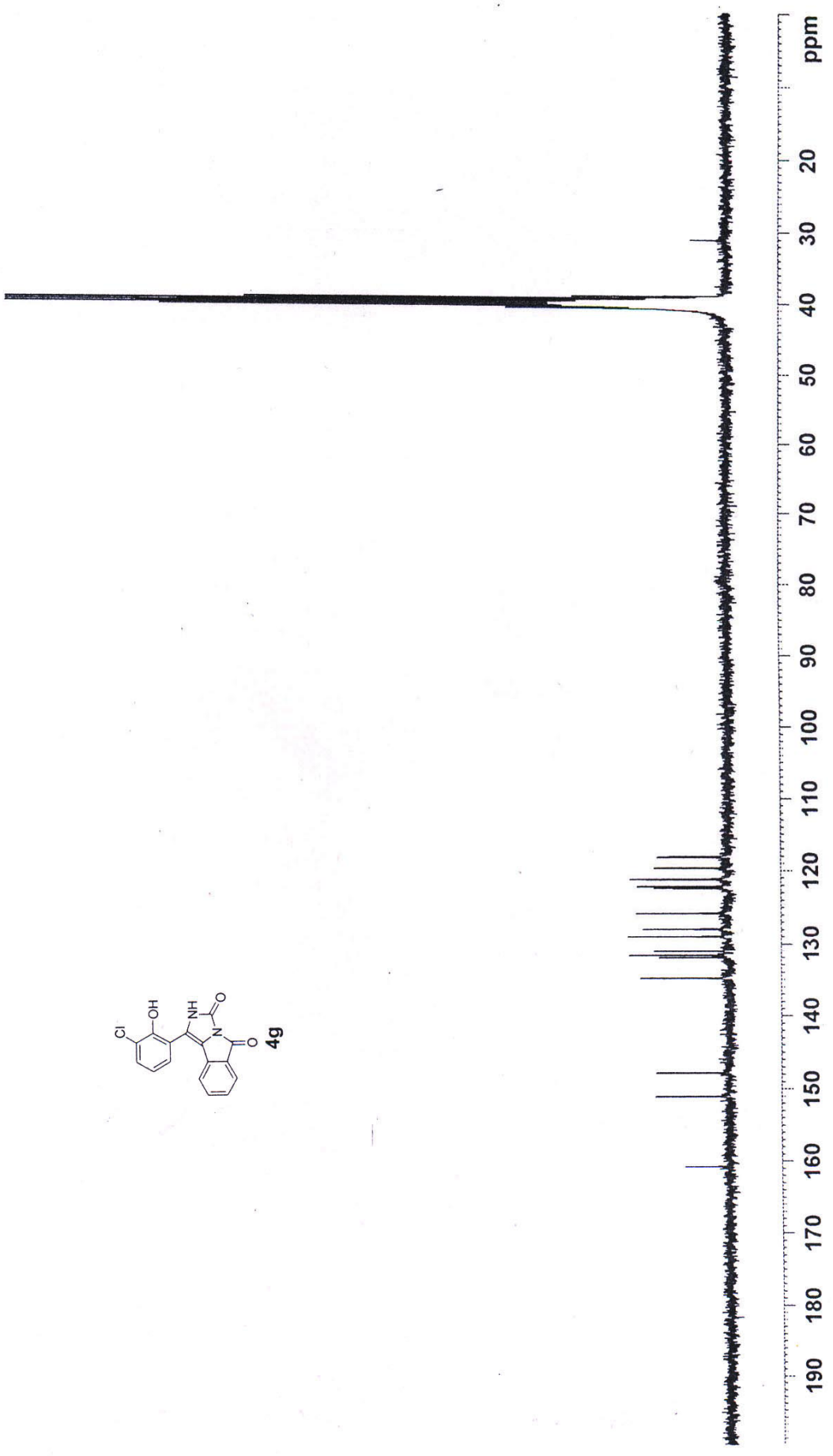




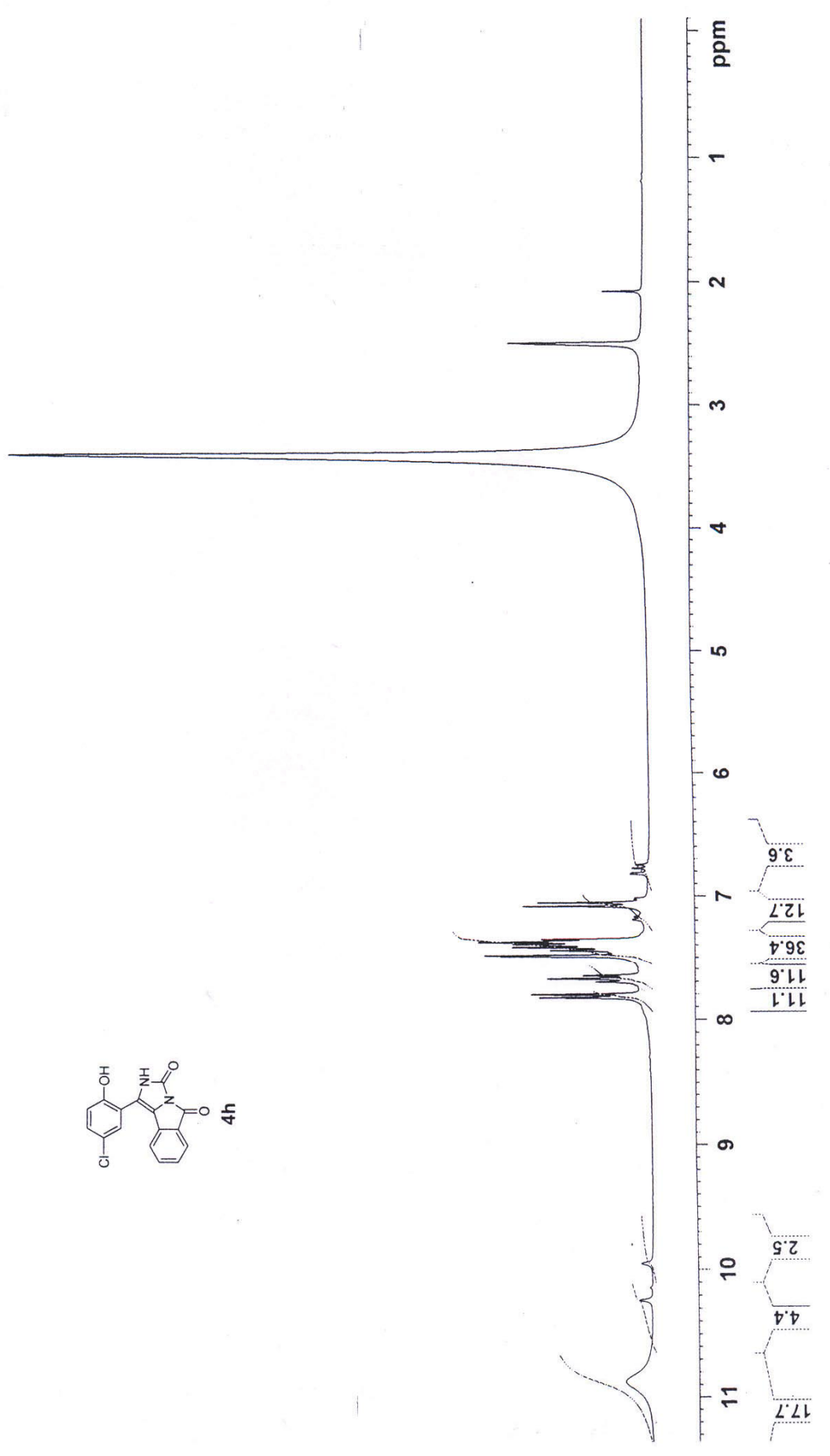



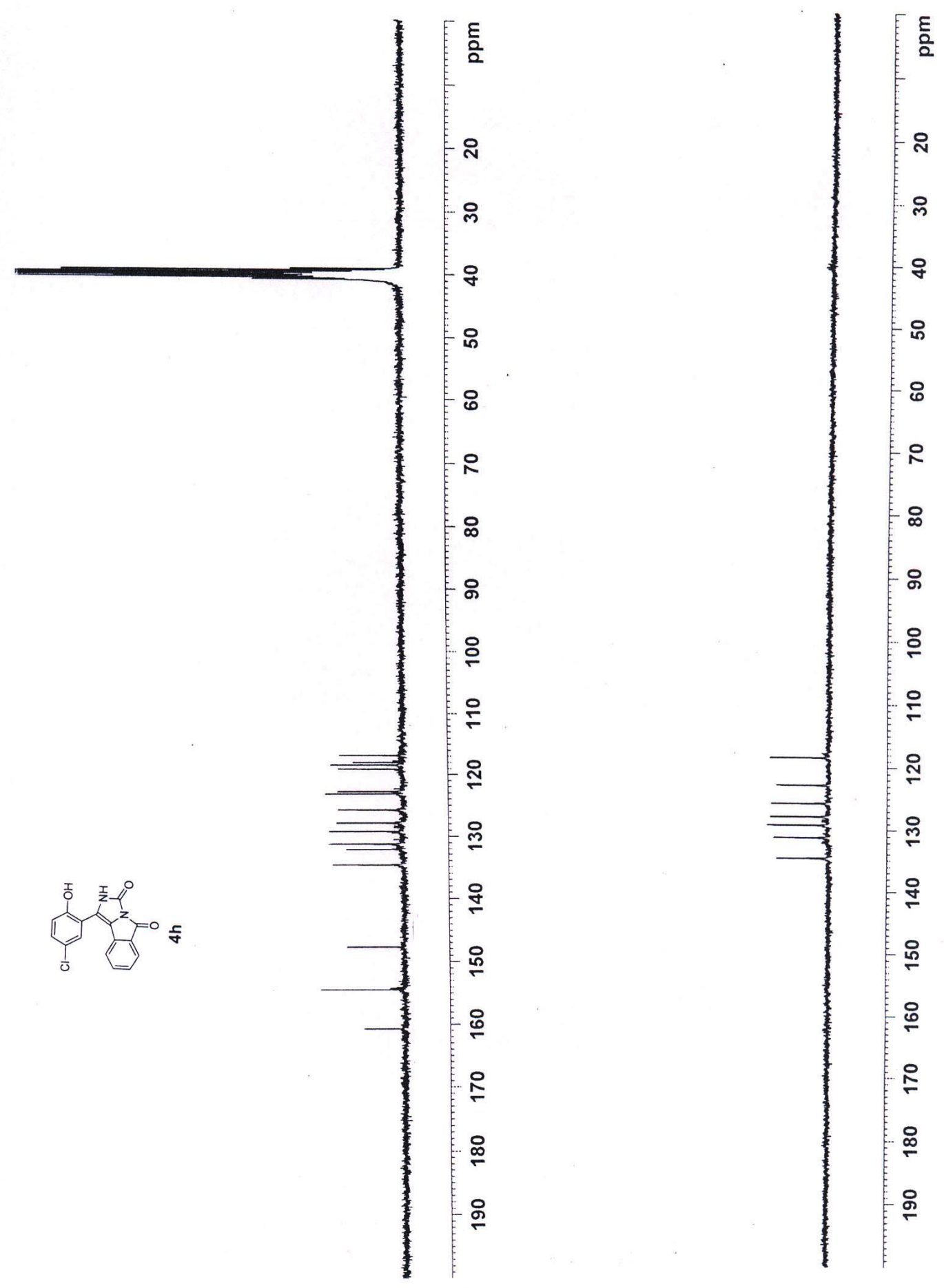


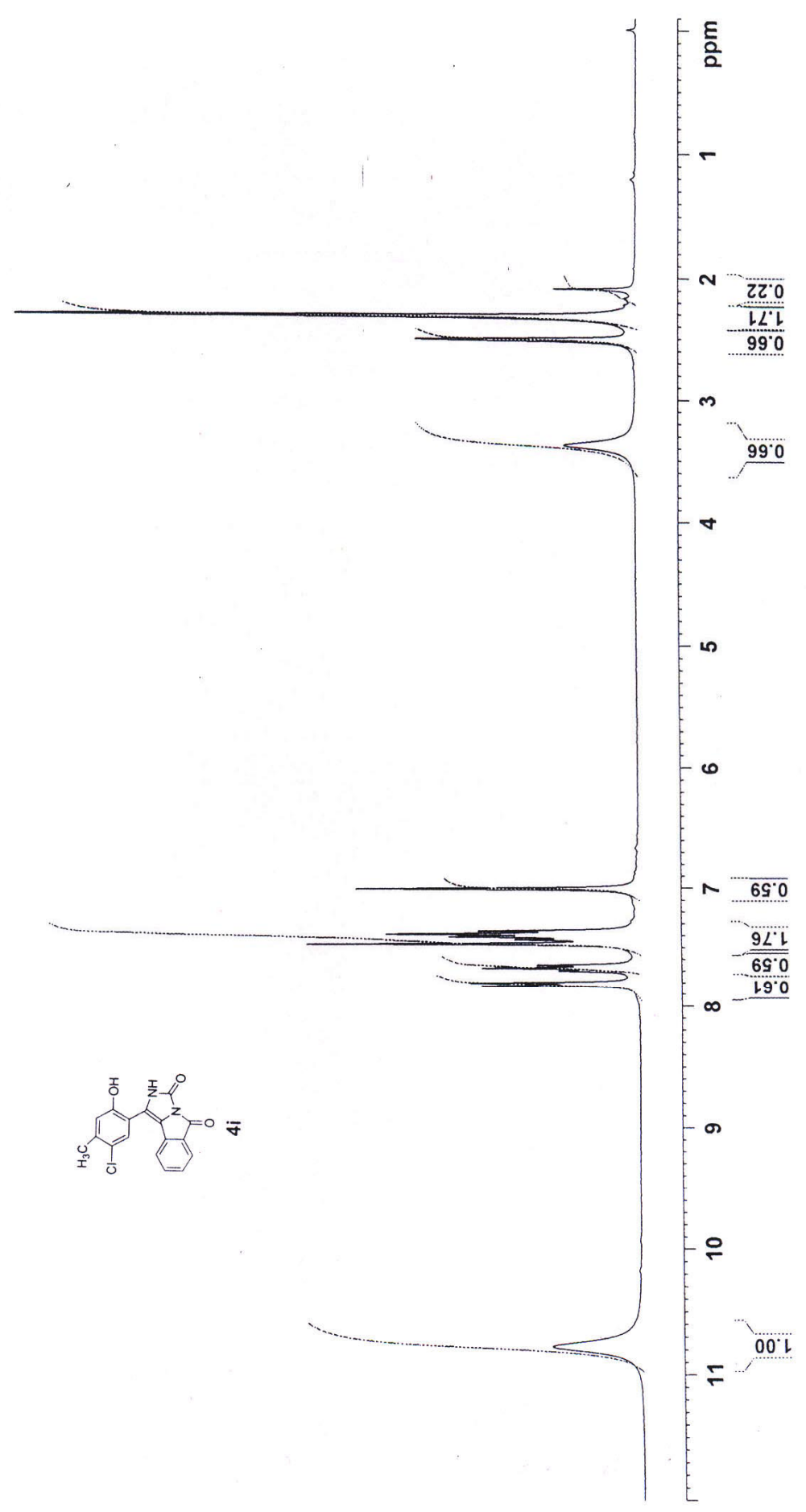




$$
\|
$$




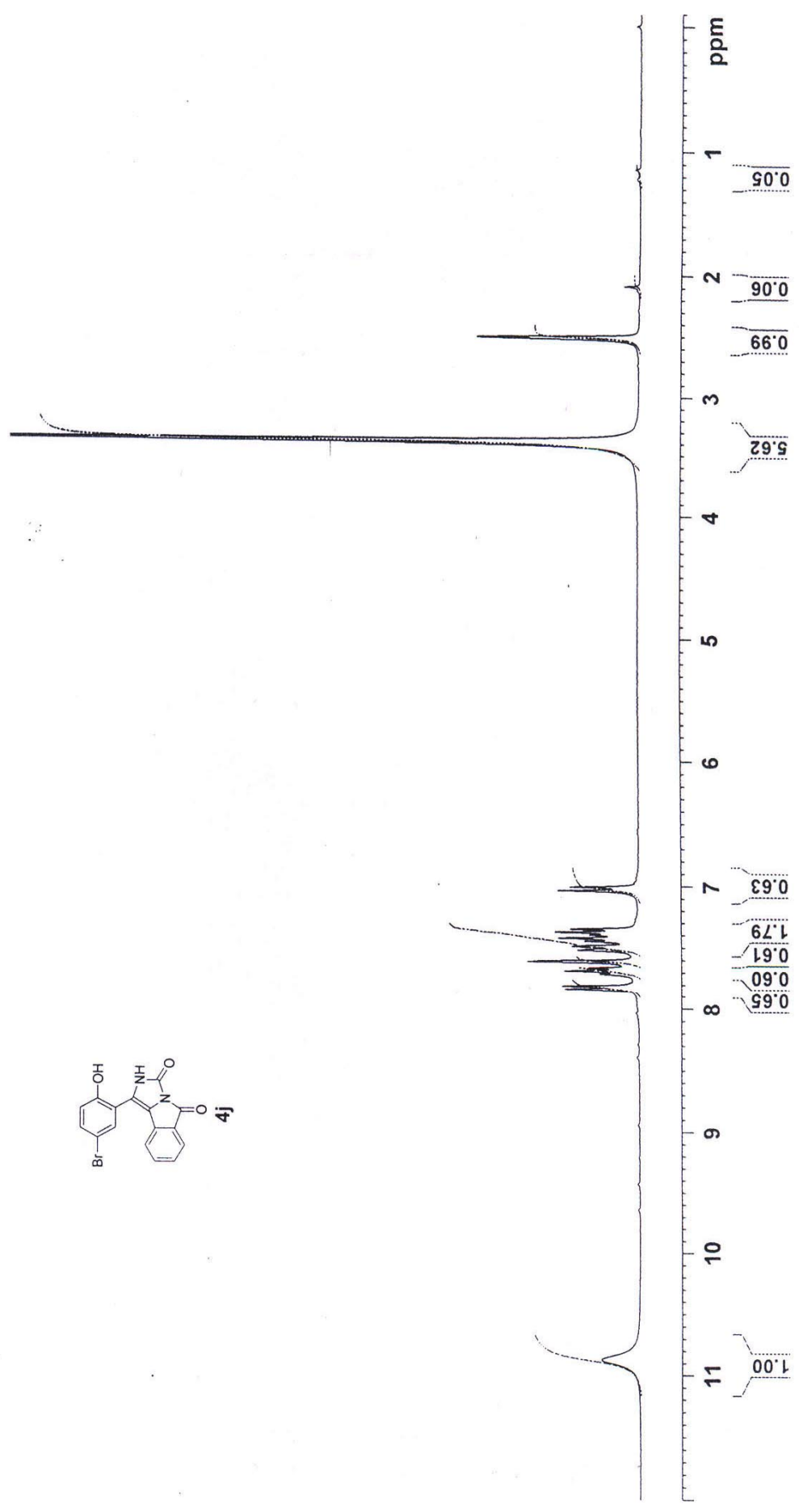



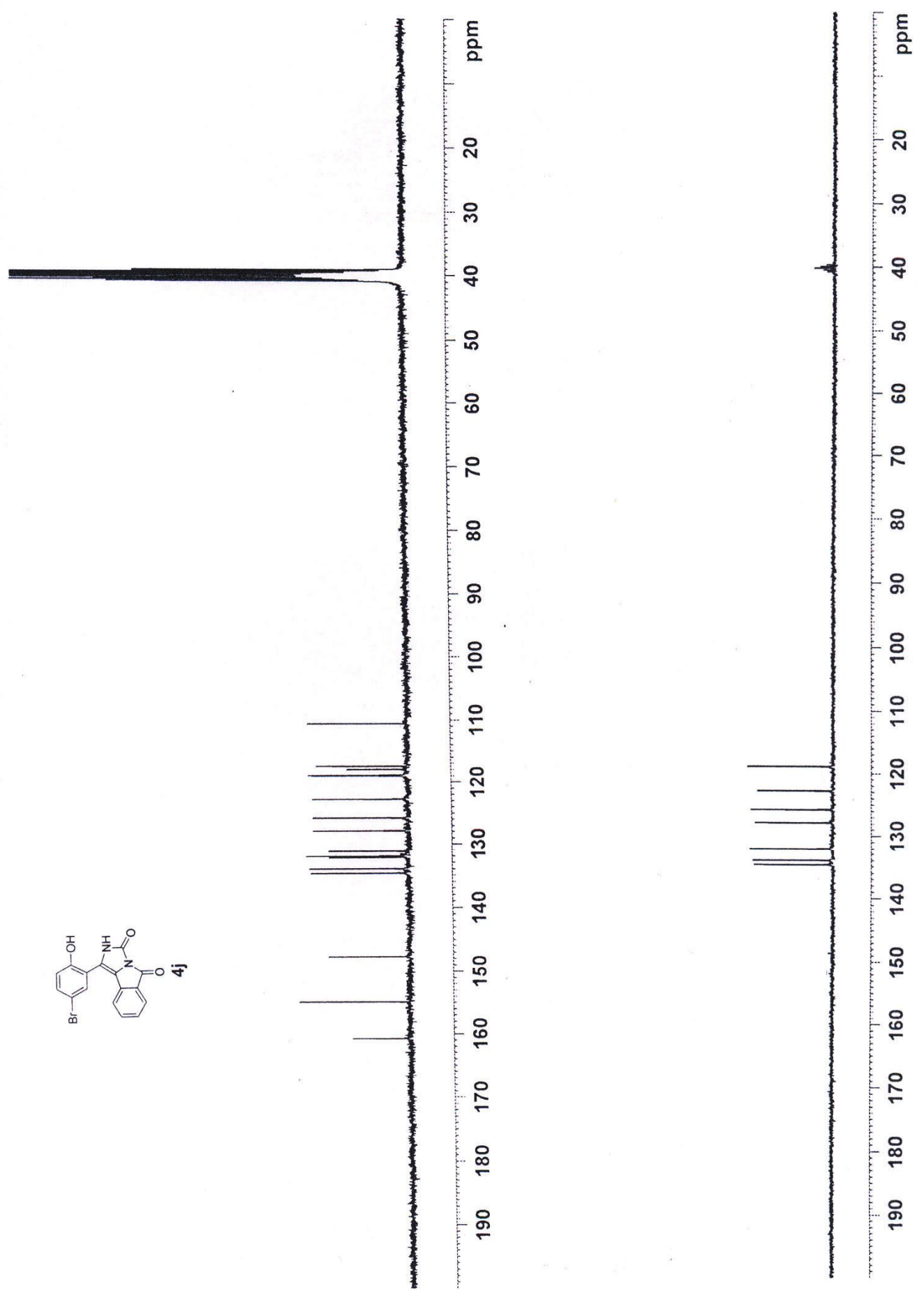


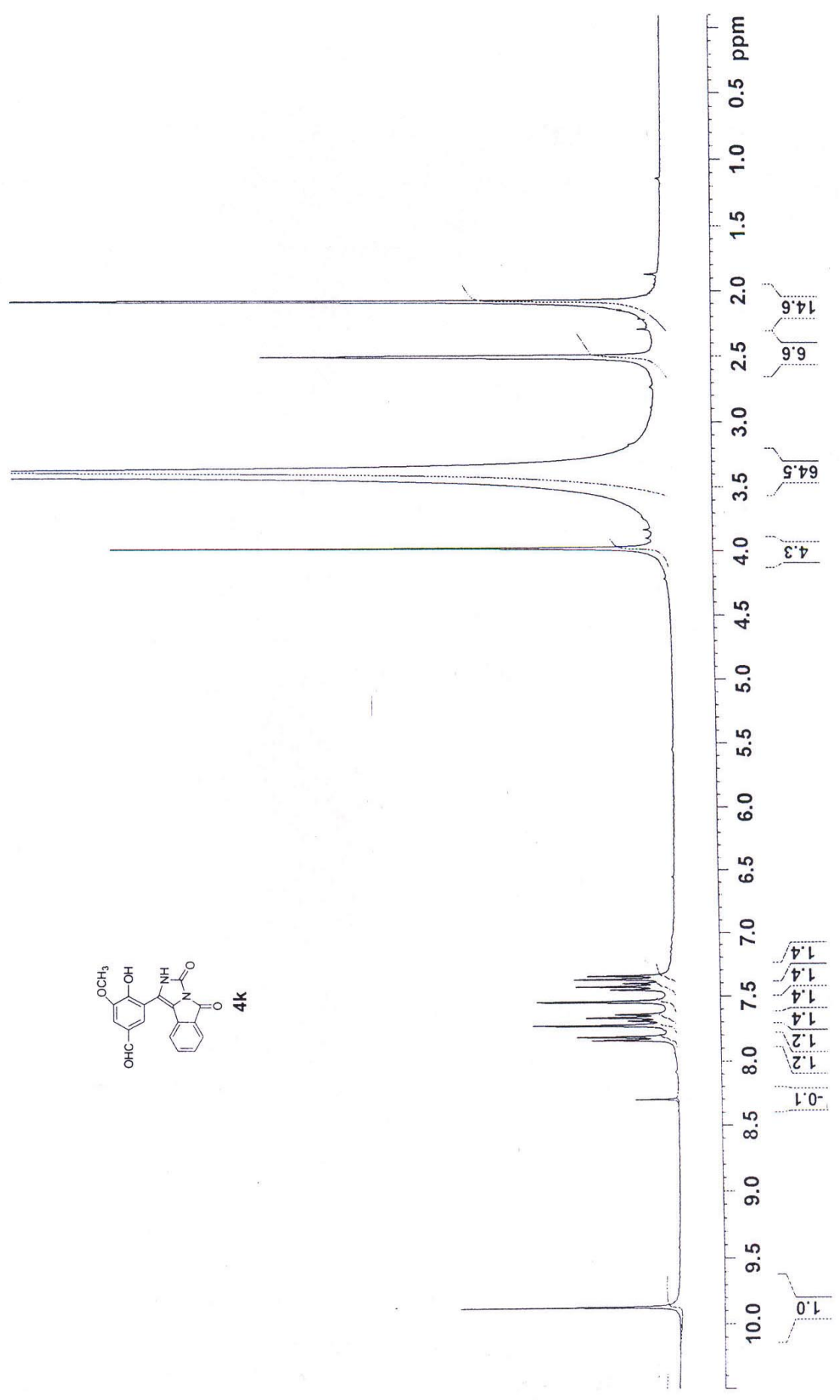




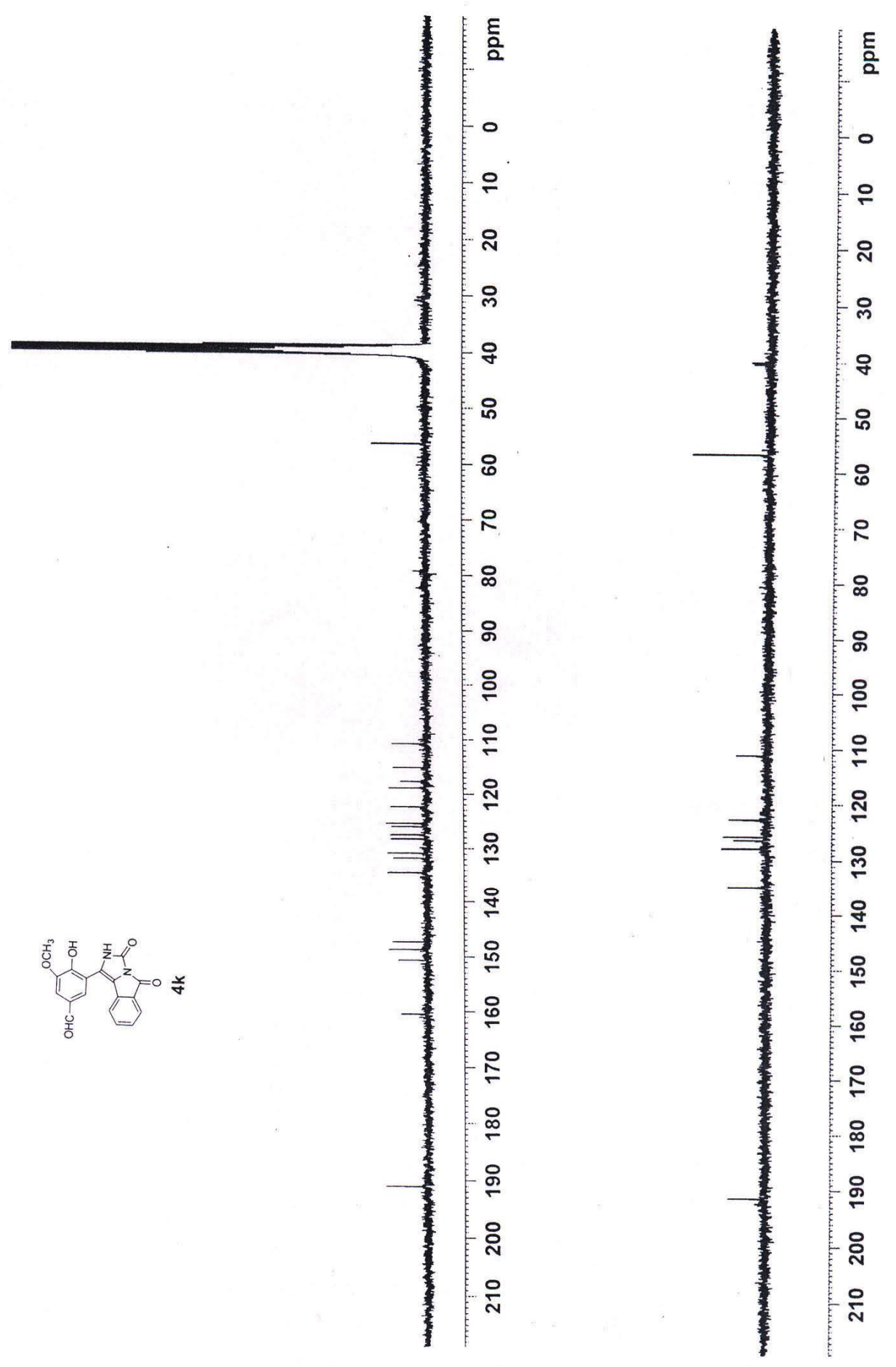




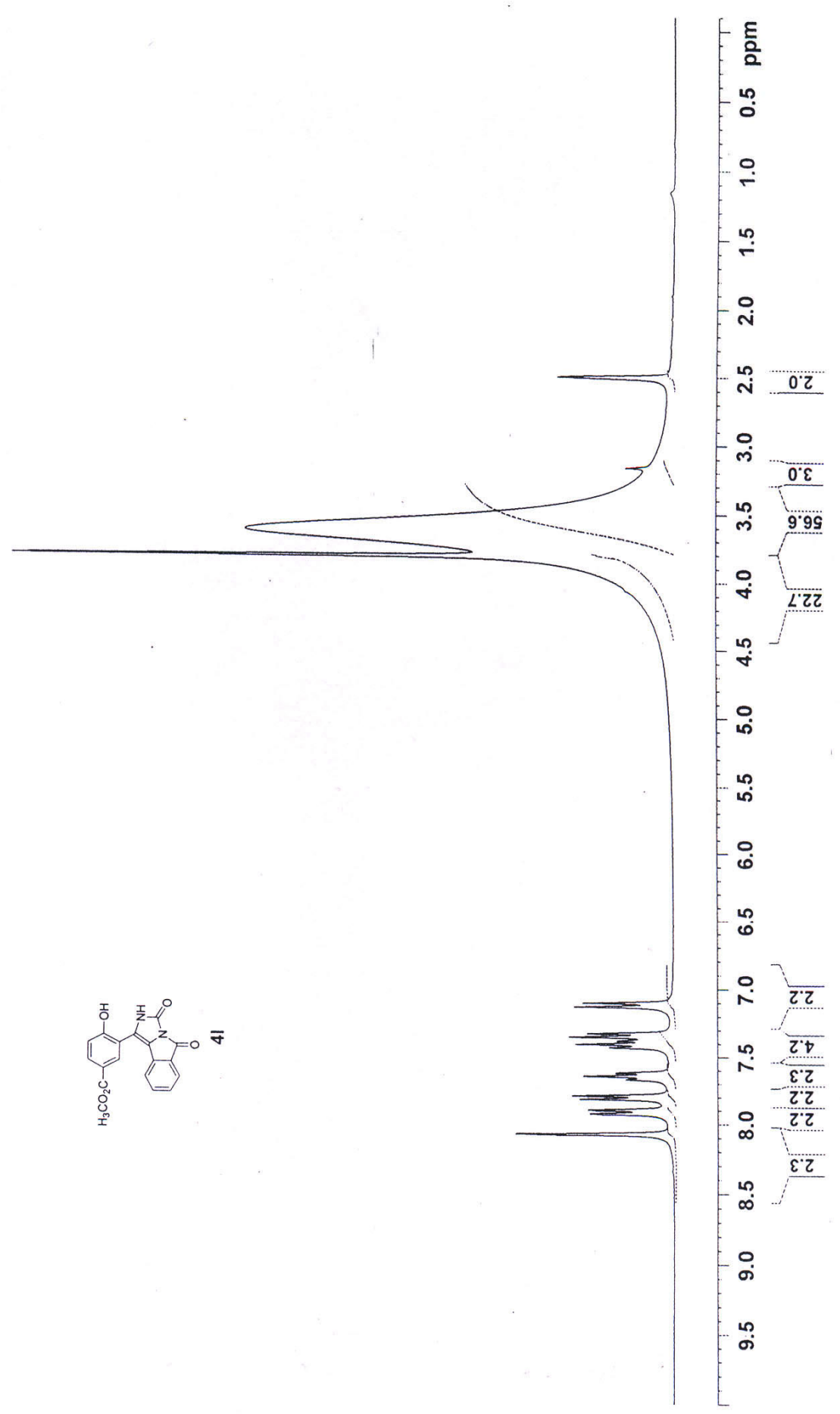



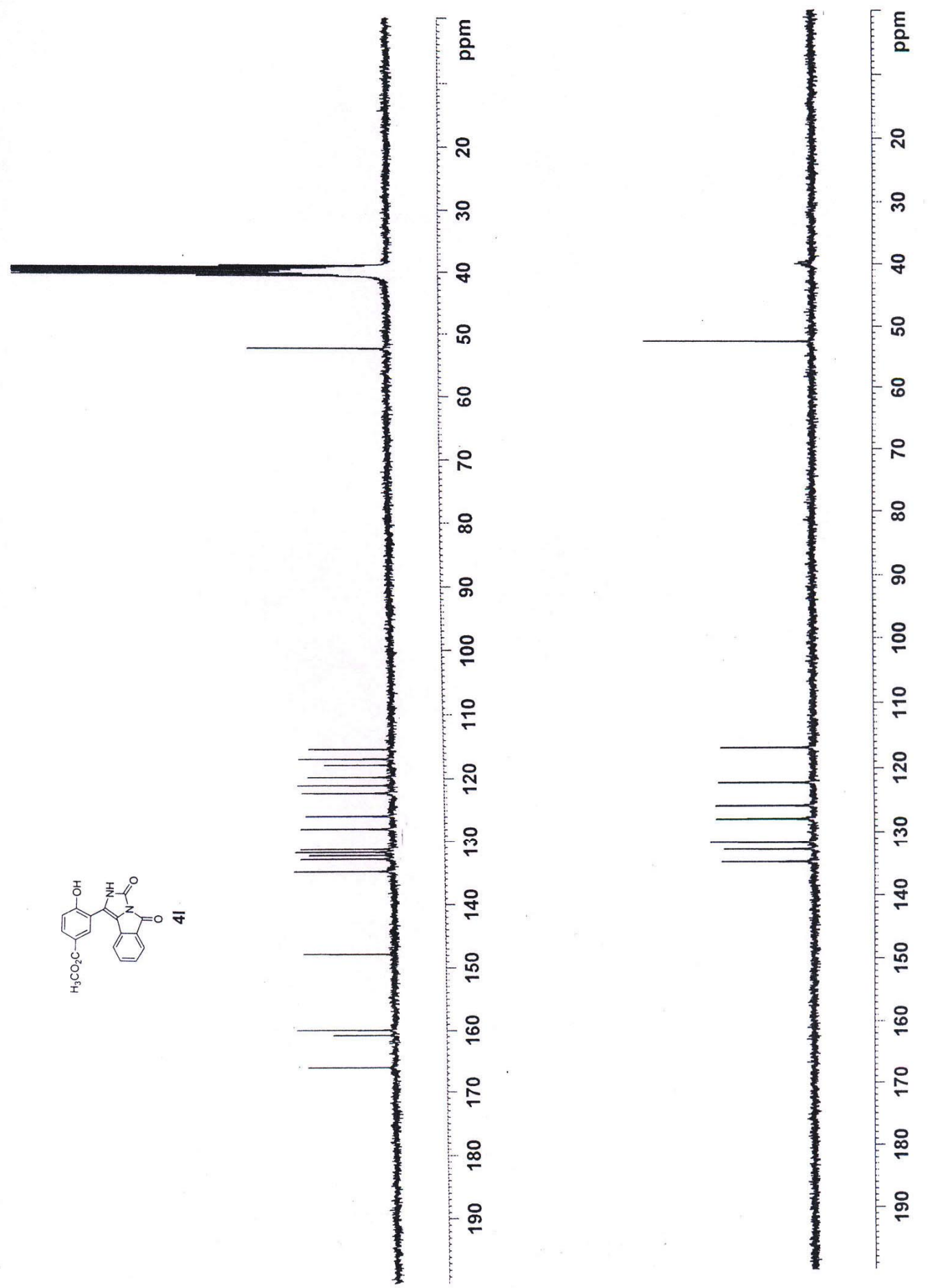


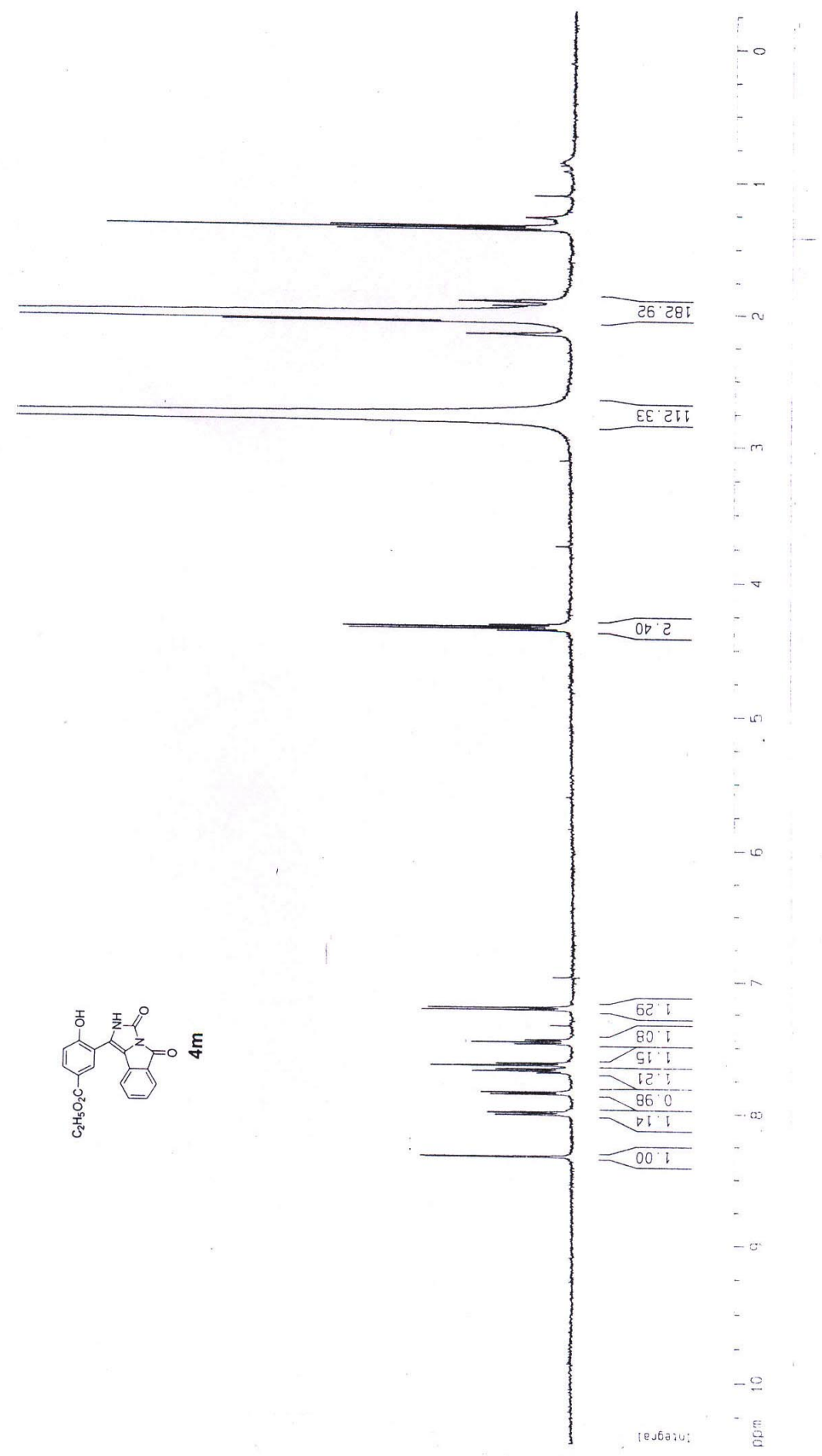




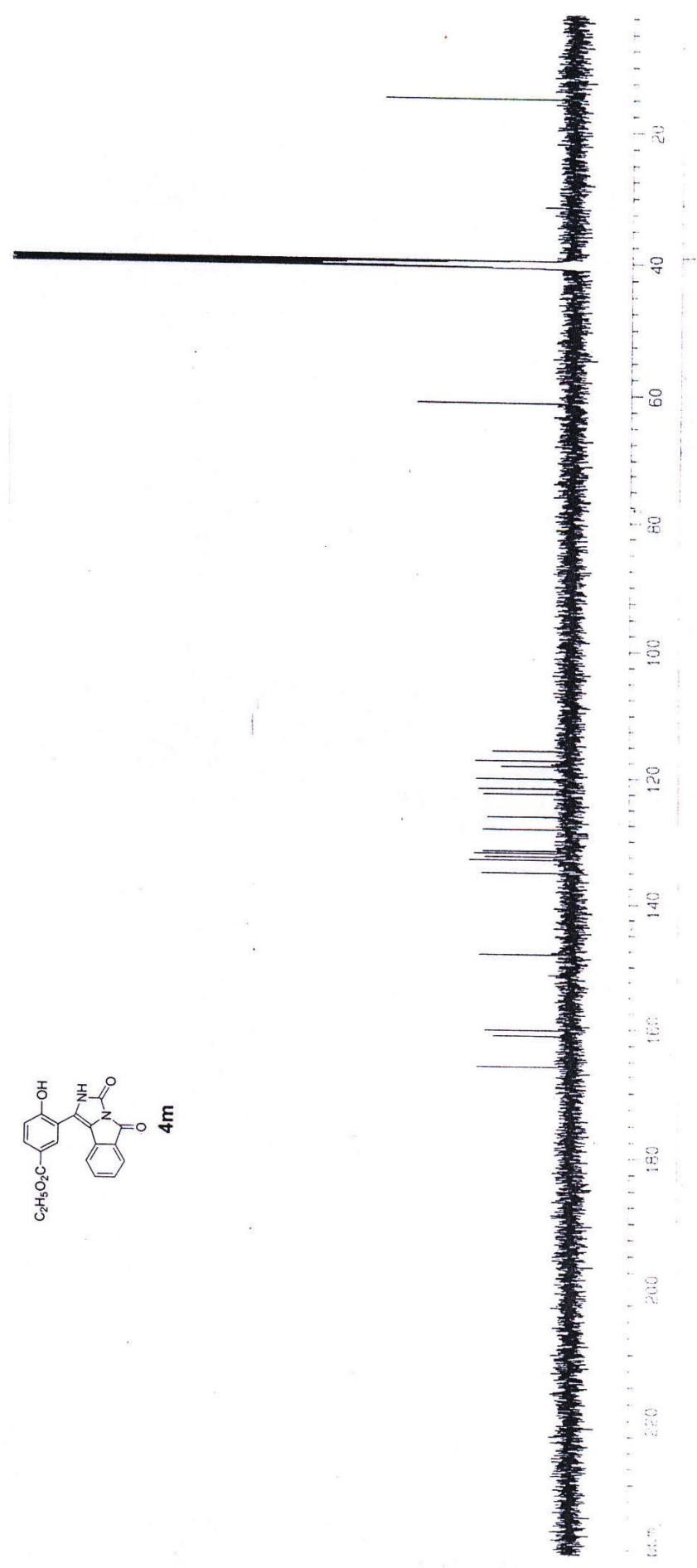




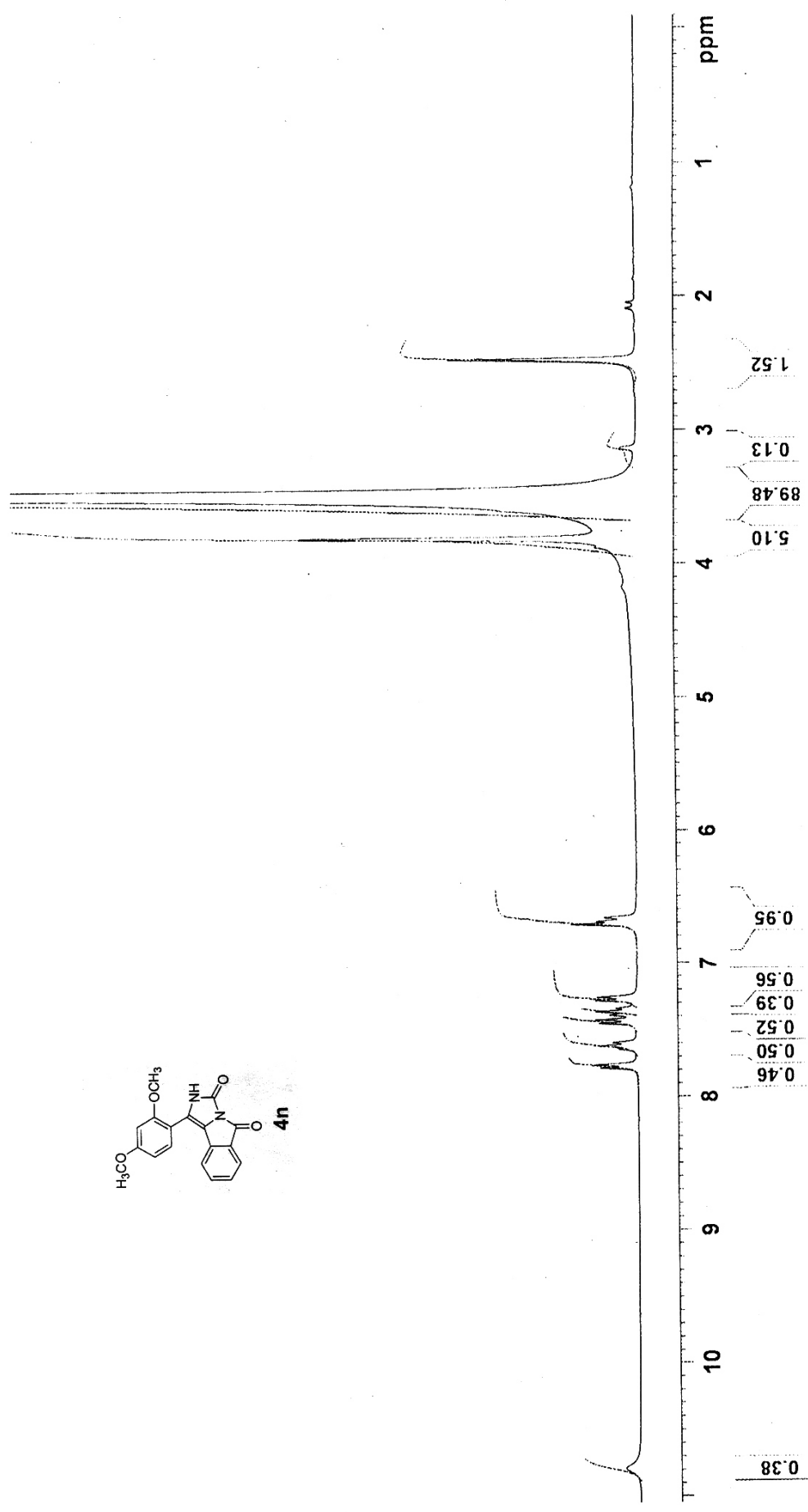



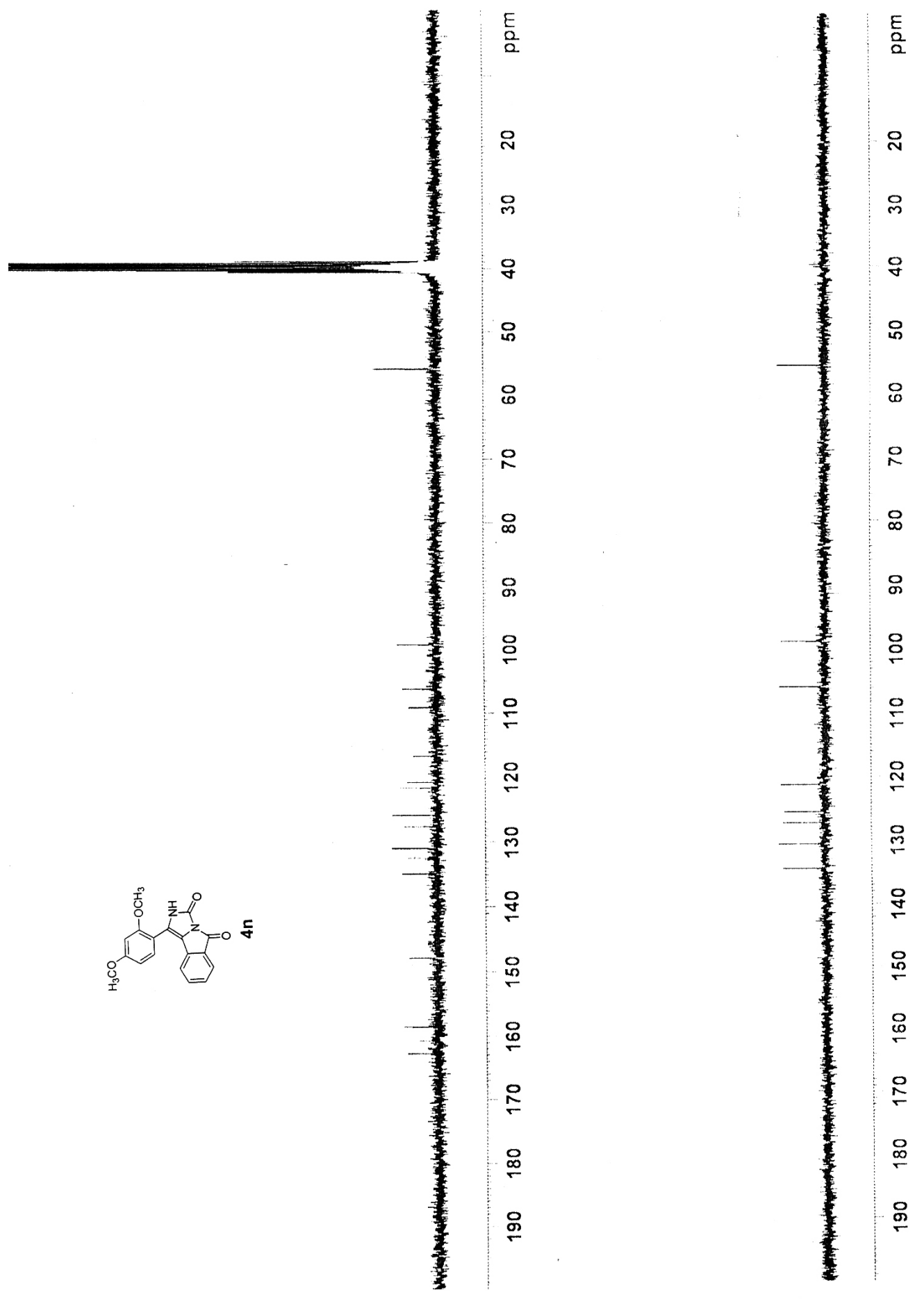


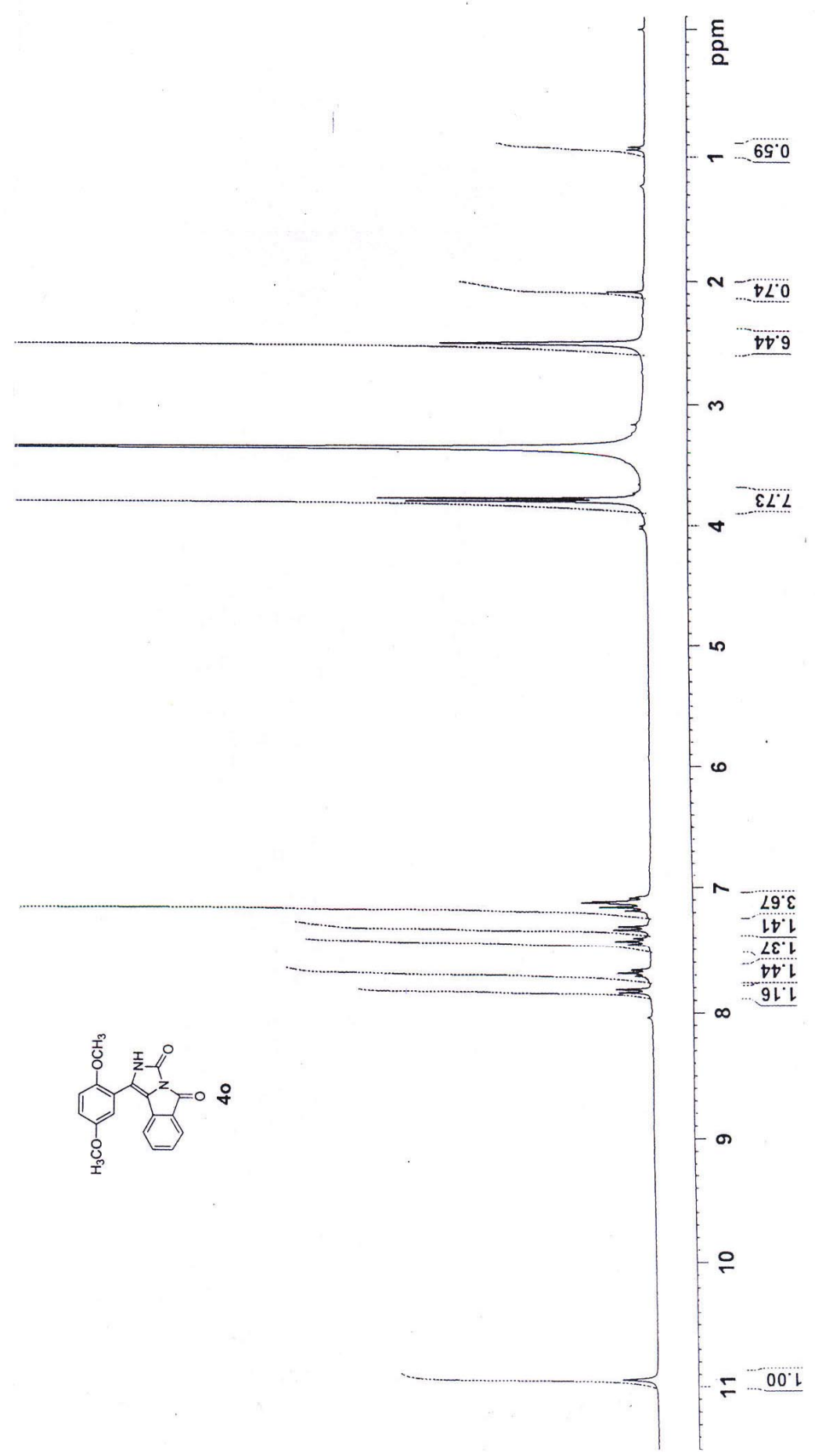



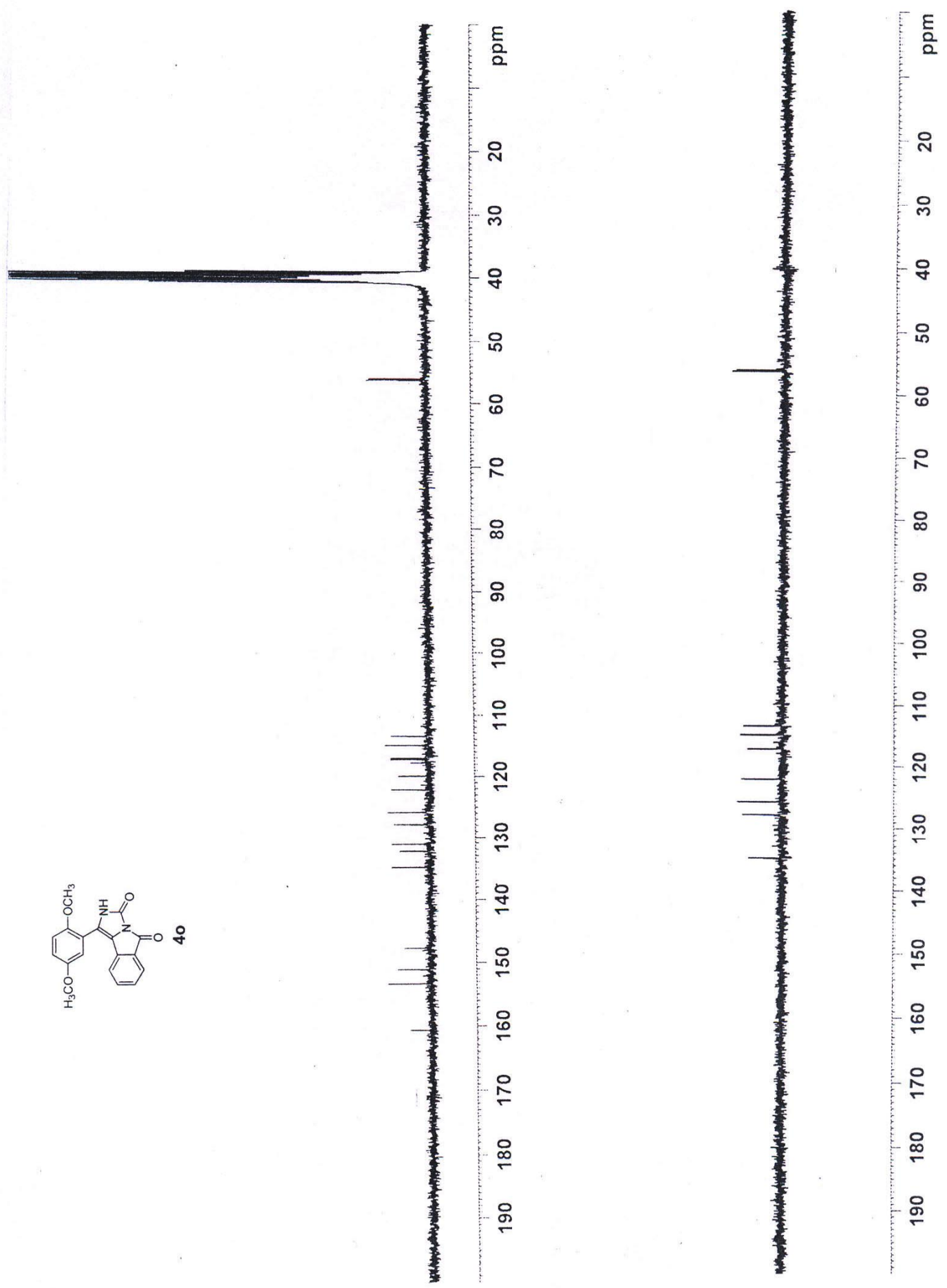

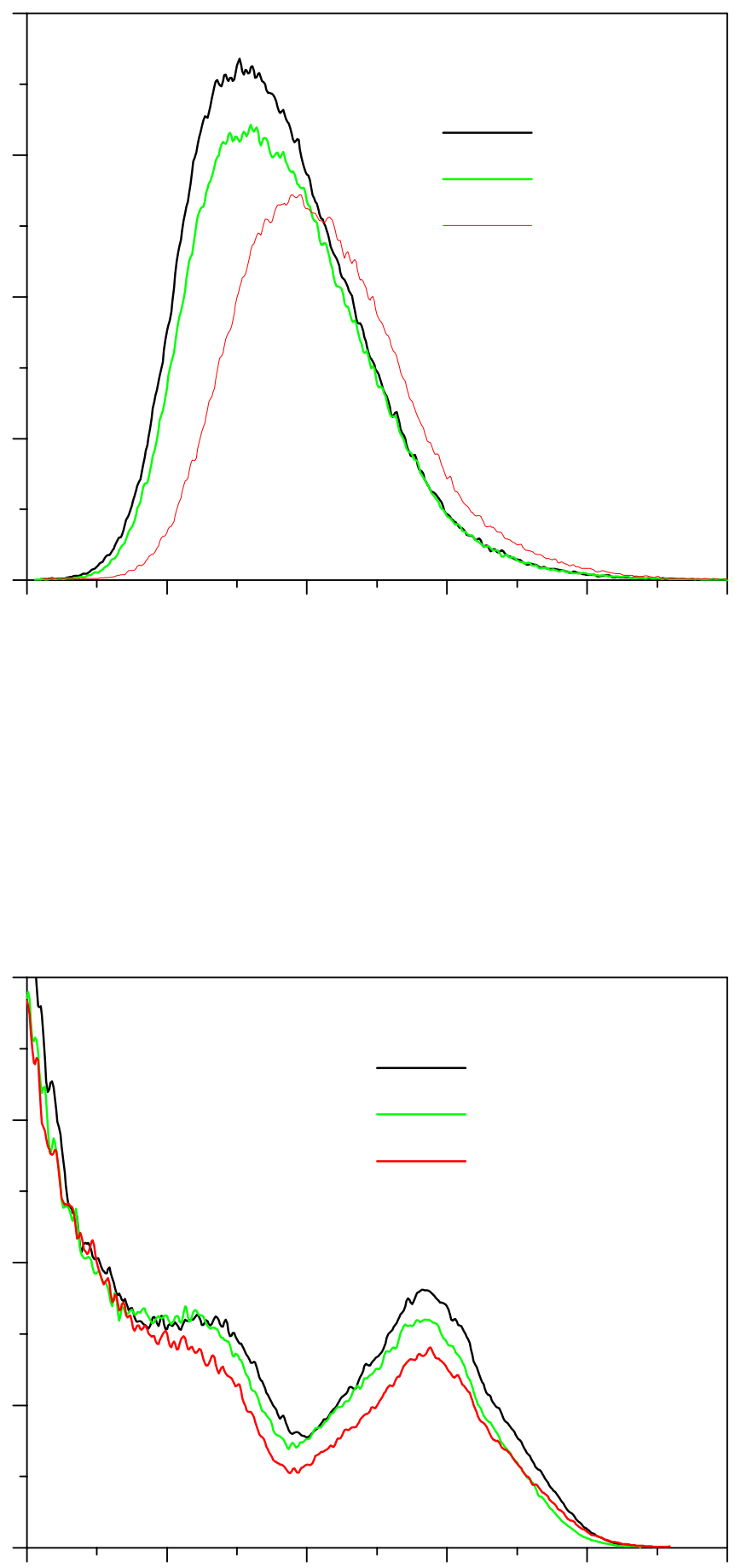

Figure 1. Fluorescence emission spectra (up) and excitation spectra (down) of $\mathbf{4 a}$ in different solvents 

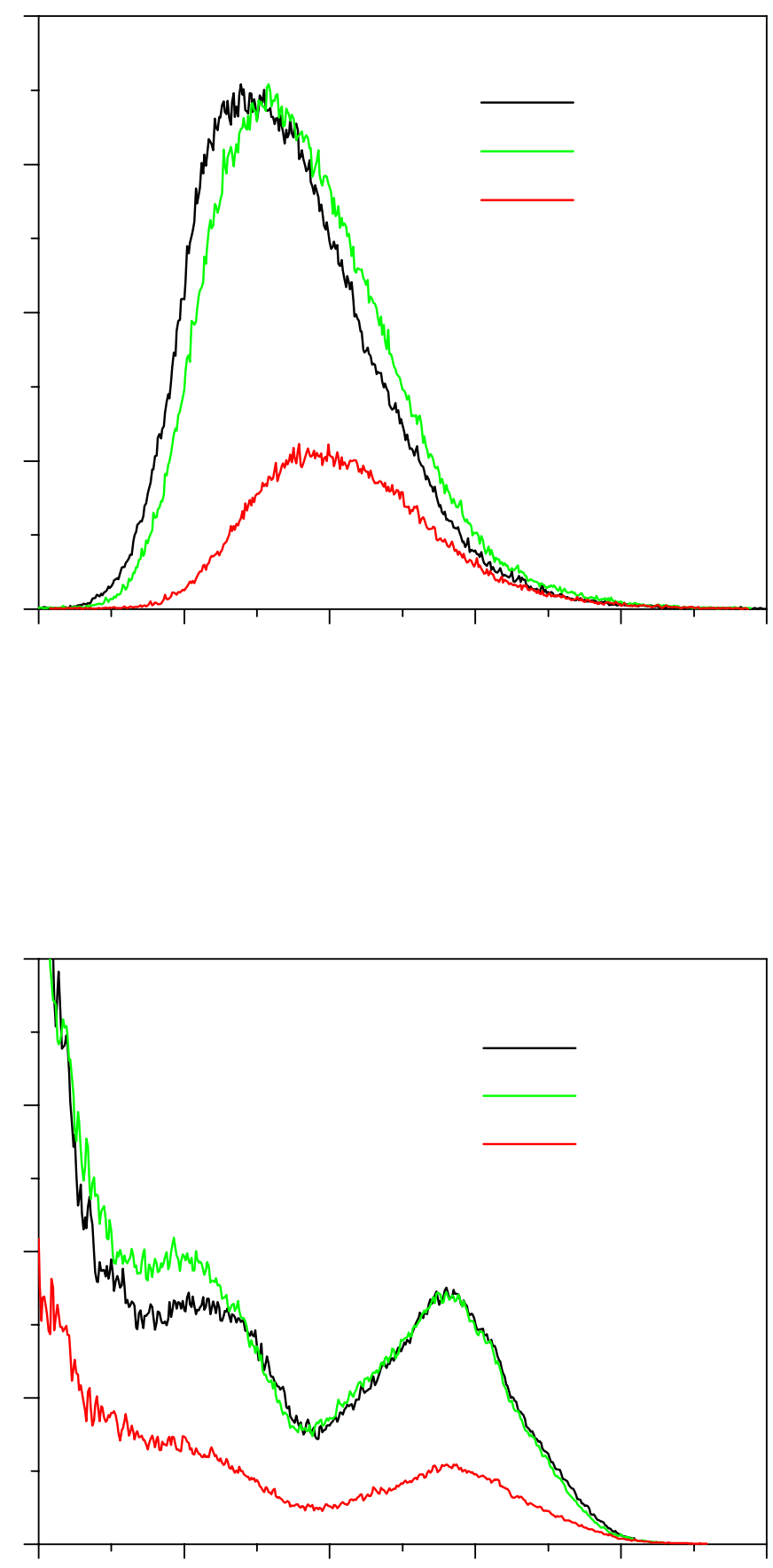

Figure 2. Fluorescence emission spectra (up) and excitation spectra (down) of $\mathbf{4 b}$ in different solvents 
39
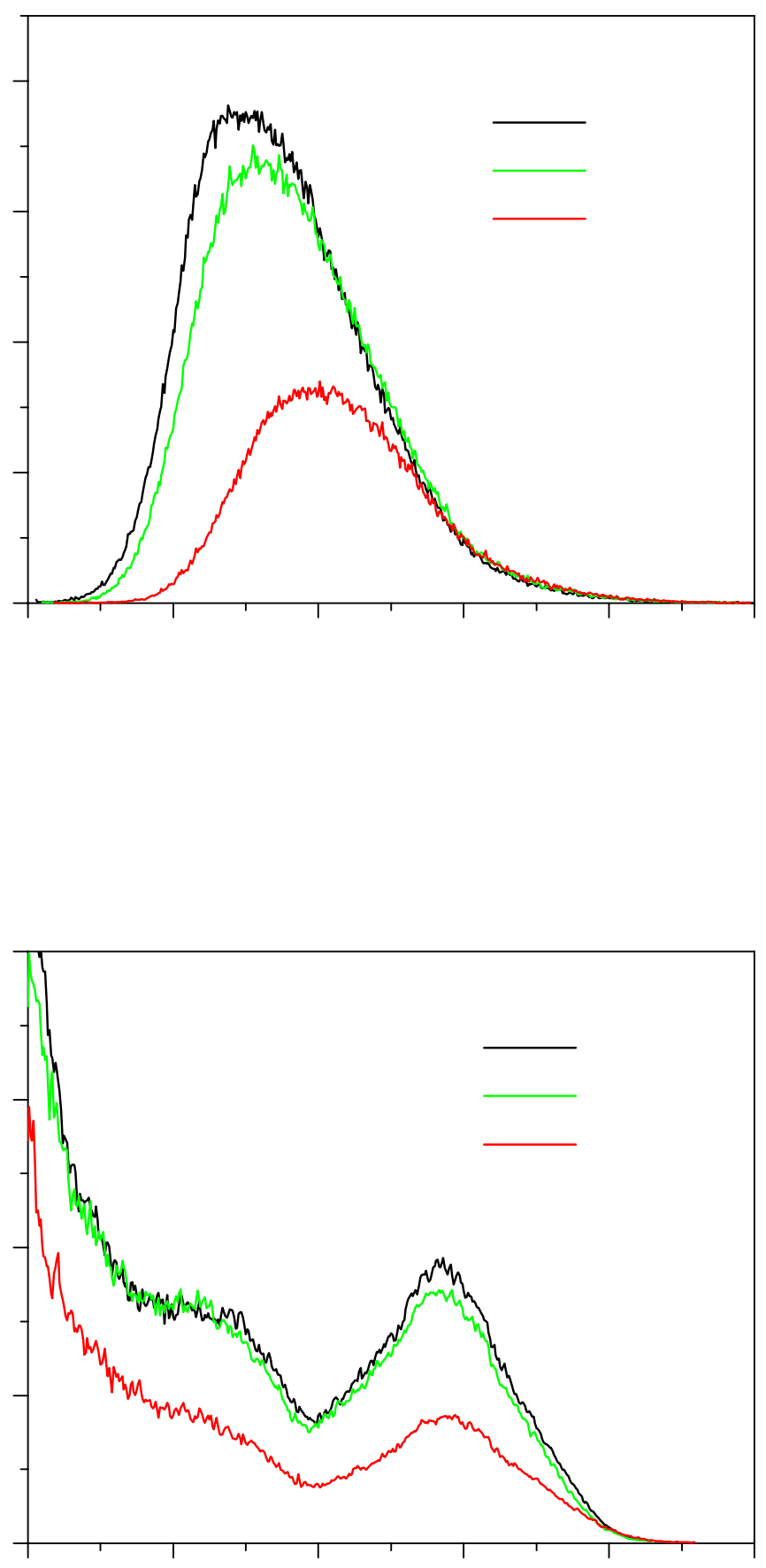

Figure 3. Fluorescence emission spectra (up) and excitation spectra (down) of $\mathbf{4 c}$ in different solvents 

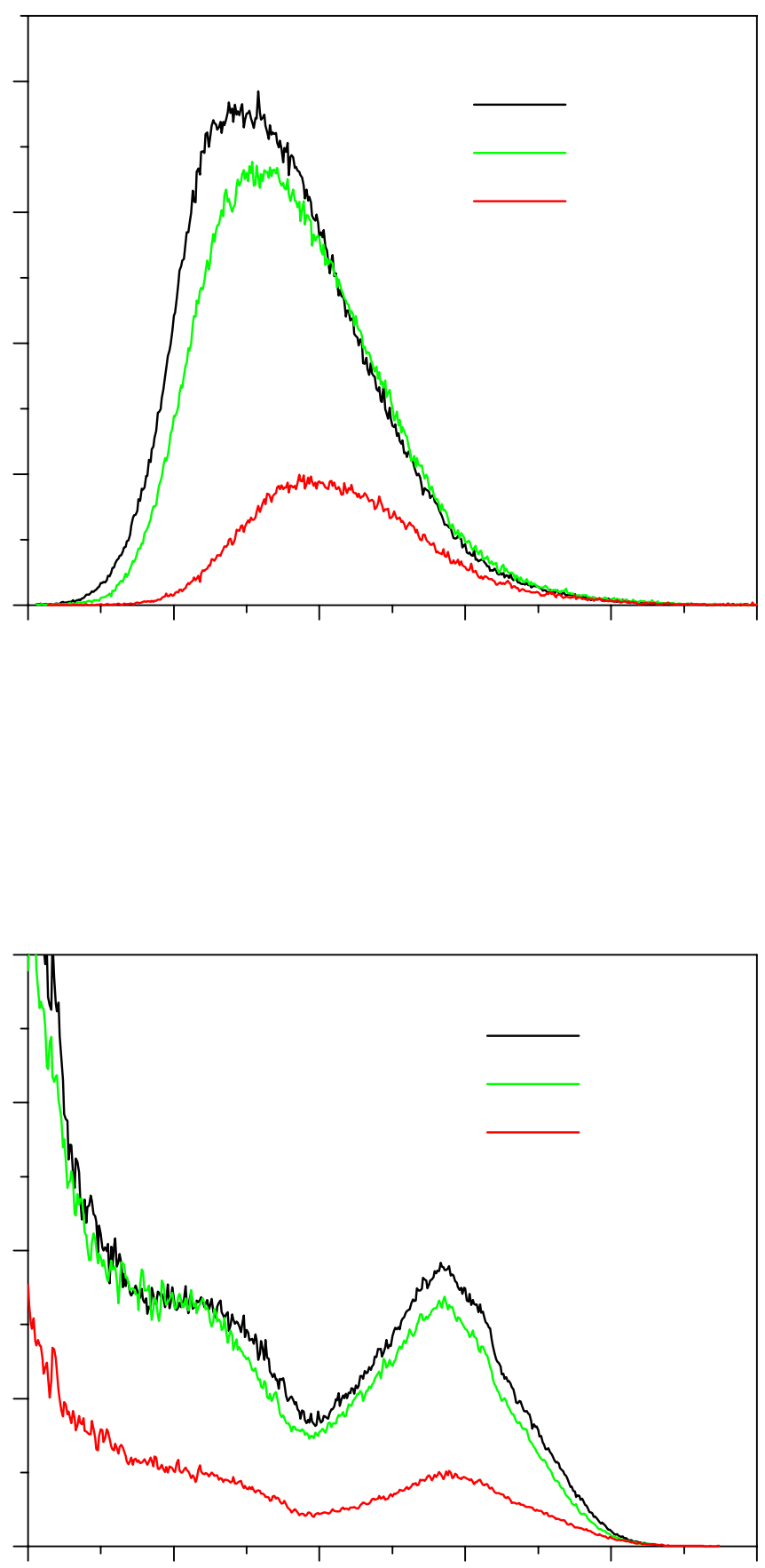

Figure 4. Fluorescence emission spectra (up) and excitation spectra (down) of 4 d in different solvents 

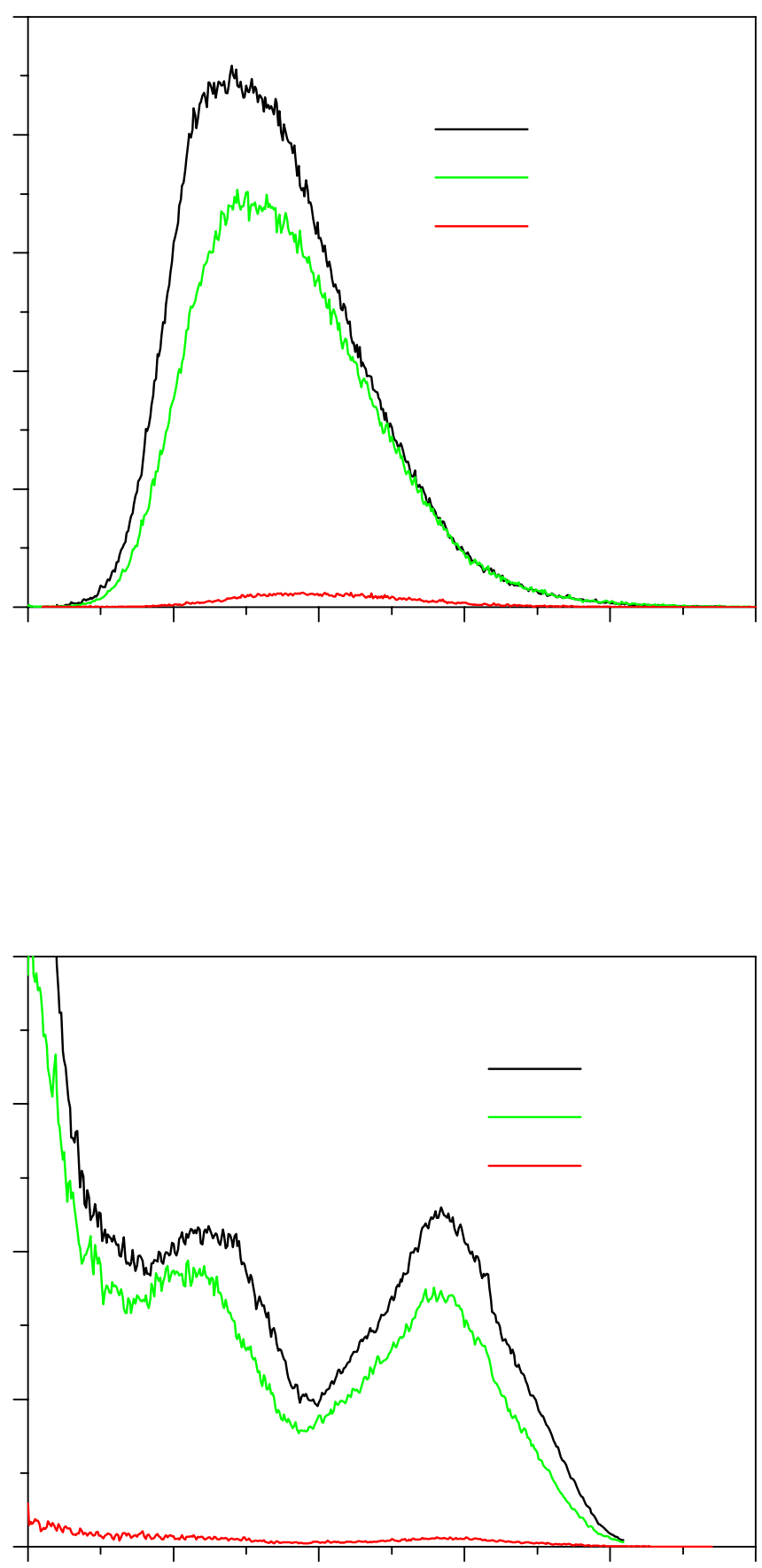

Figure 5. Fluorescence emission spectra (up) and excitation spectra (down) of $\mathbf{4 e}$ in different solvents 

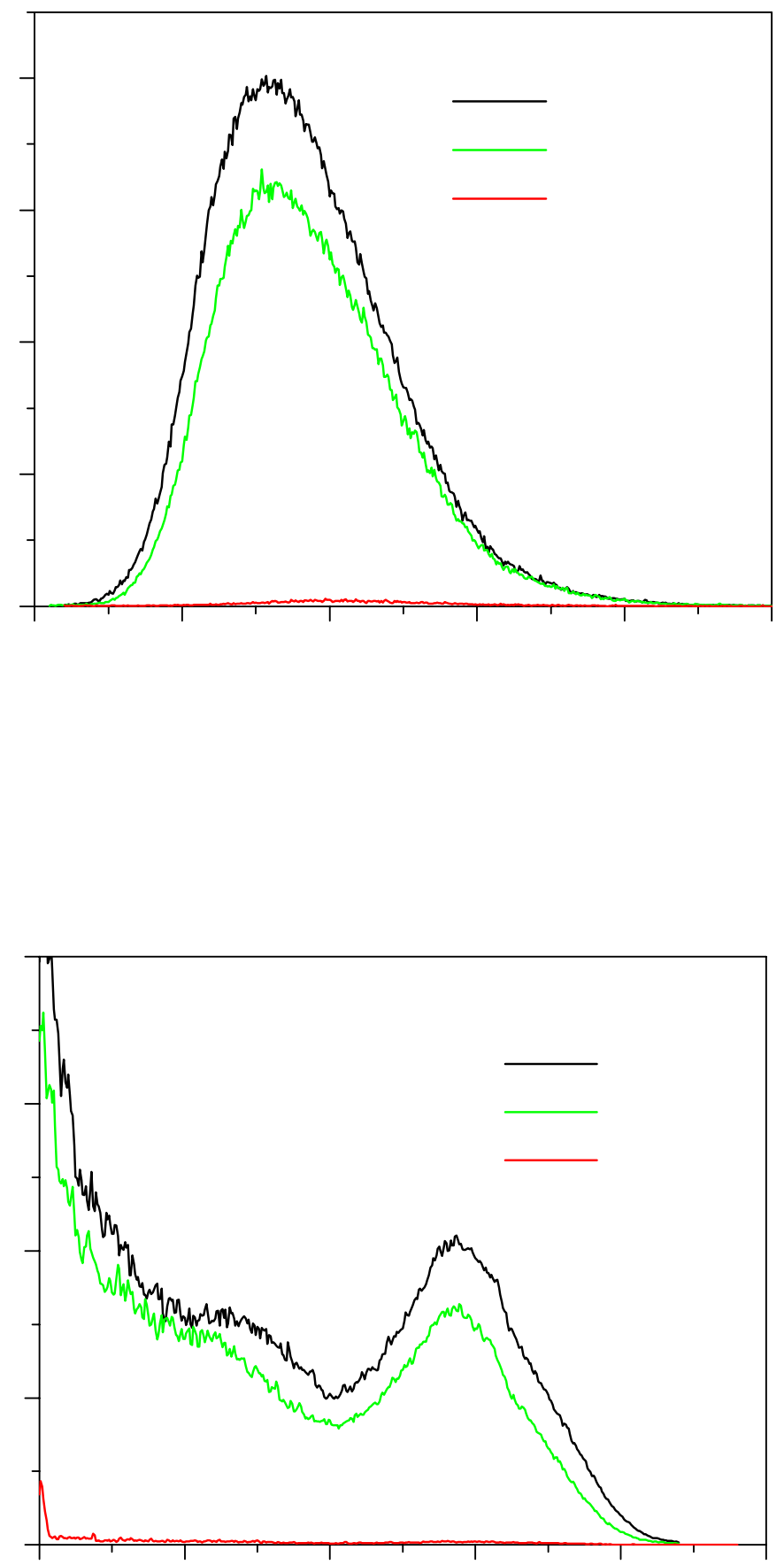

Figure 6. Fluorescence emission spectra (up) and excitation spectra (down) of $\mathbf{4 f}$ in different solvents 

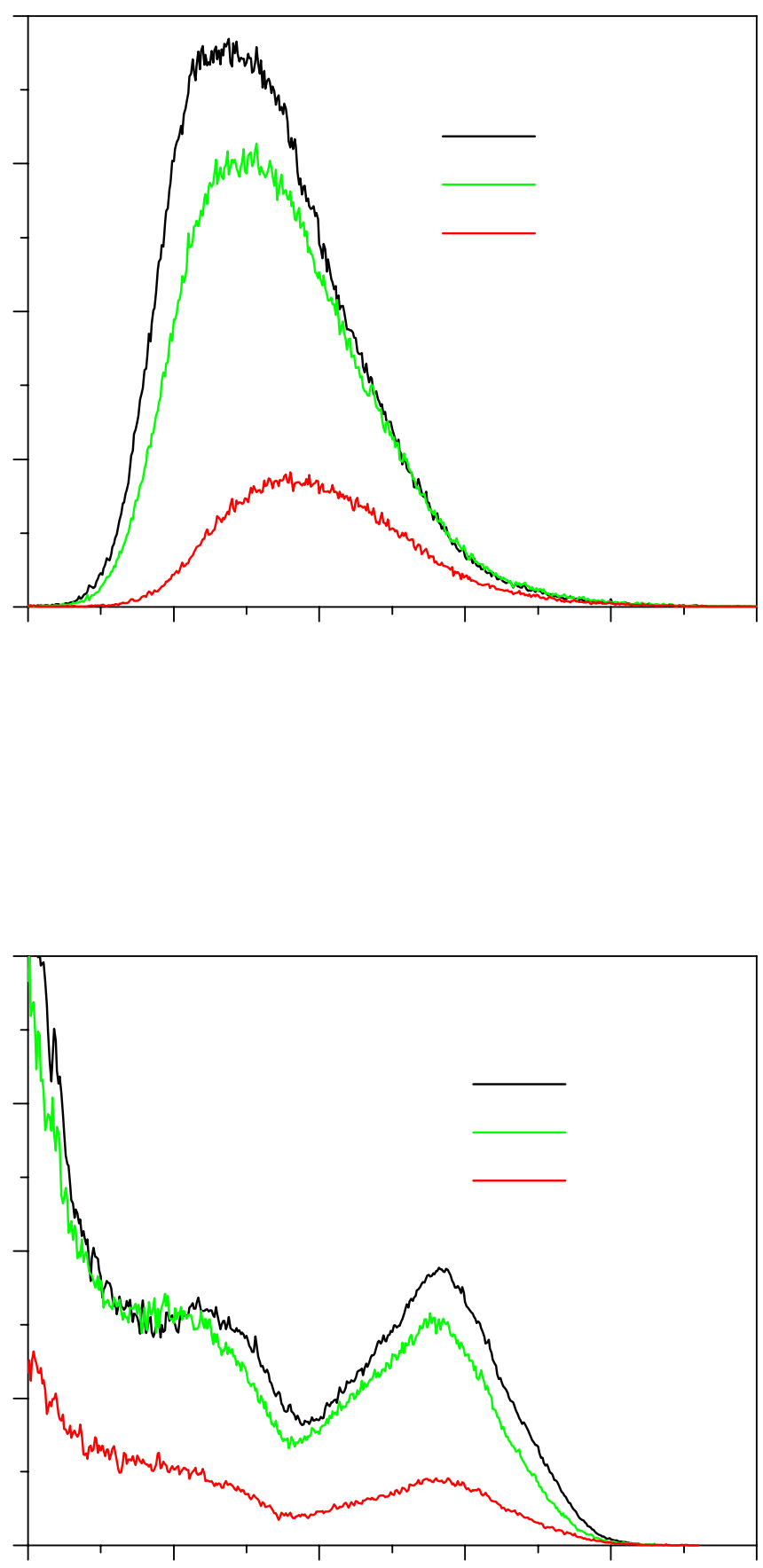

Figure 7. Fluorescence emission spectra (up) and excitation spectra (down) of $\mathbf{4 g}$ in different solvents 

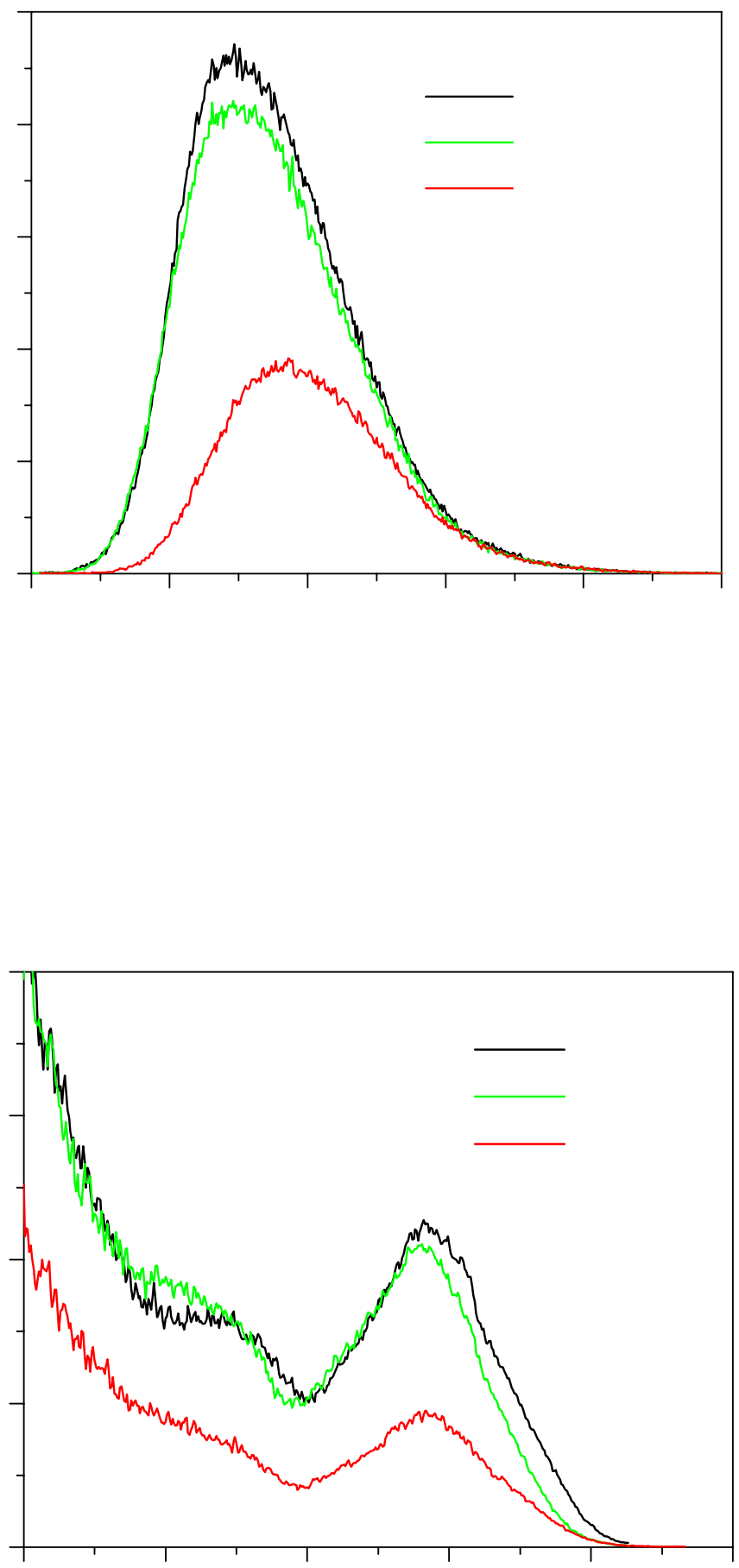

Figure 8. Fluorescence emission spectra (up) and excitation spectra (down) of $\mathbf{4 h}$ in different solvents 

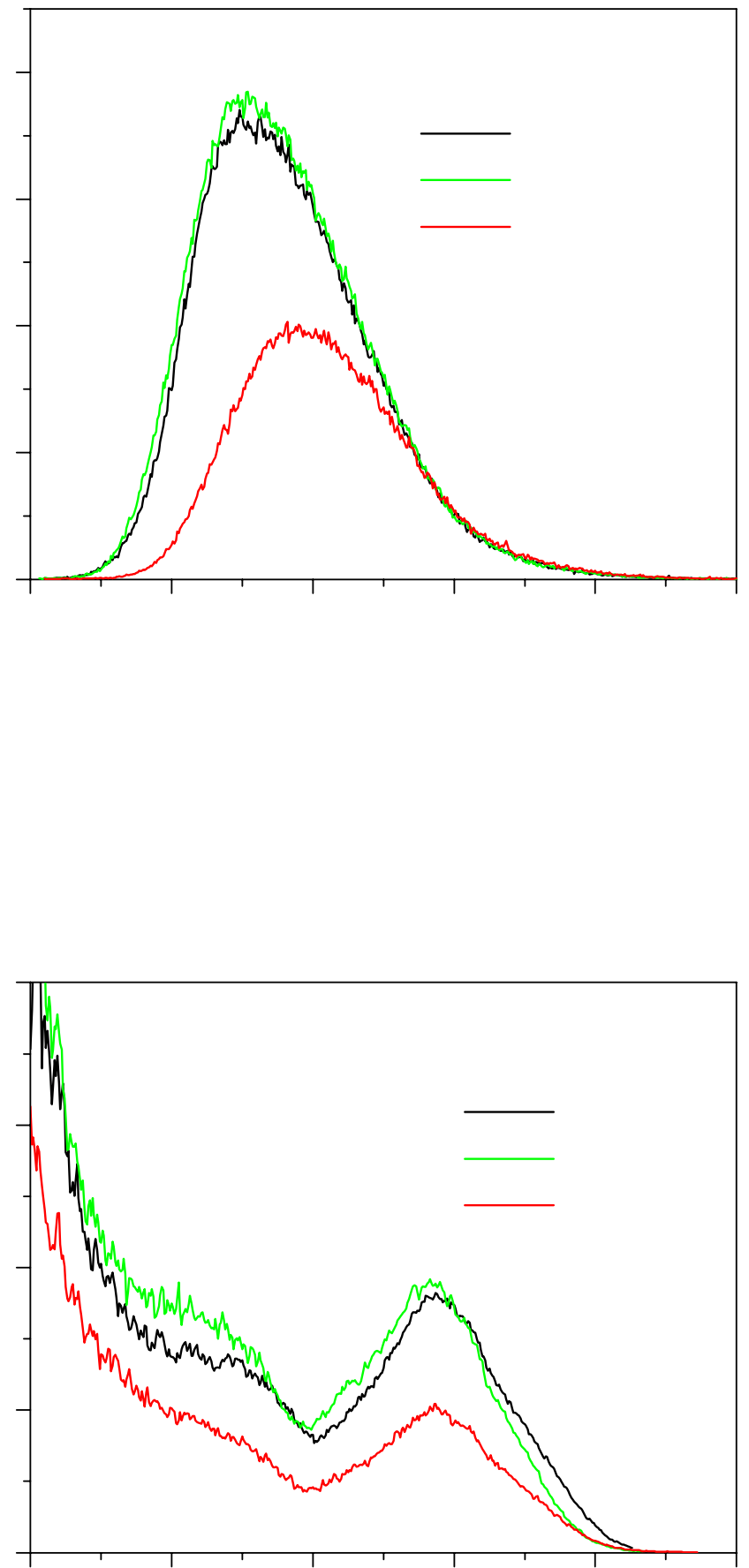

Figure 9. Fluorescence emission spectra (up) and excitation spectra (down) of $\mathbf{4 i}$ in different solvents 

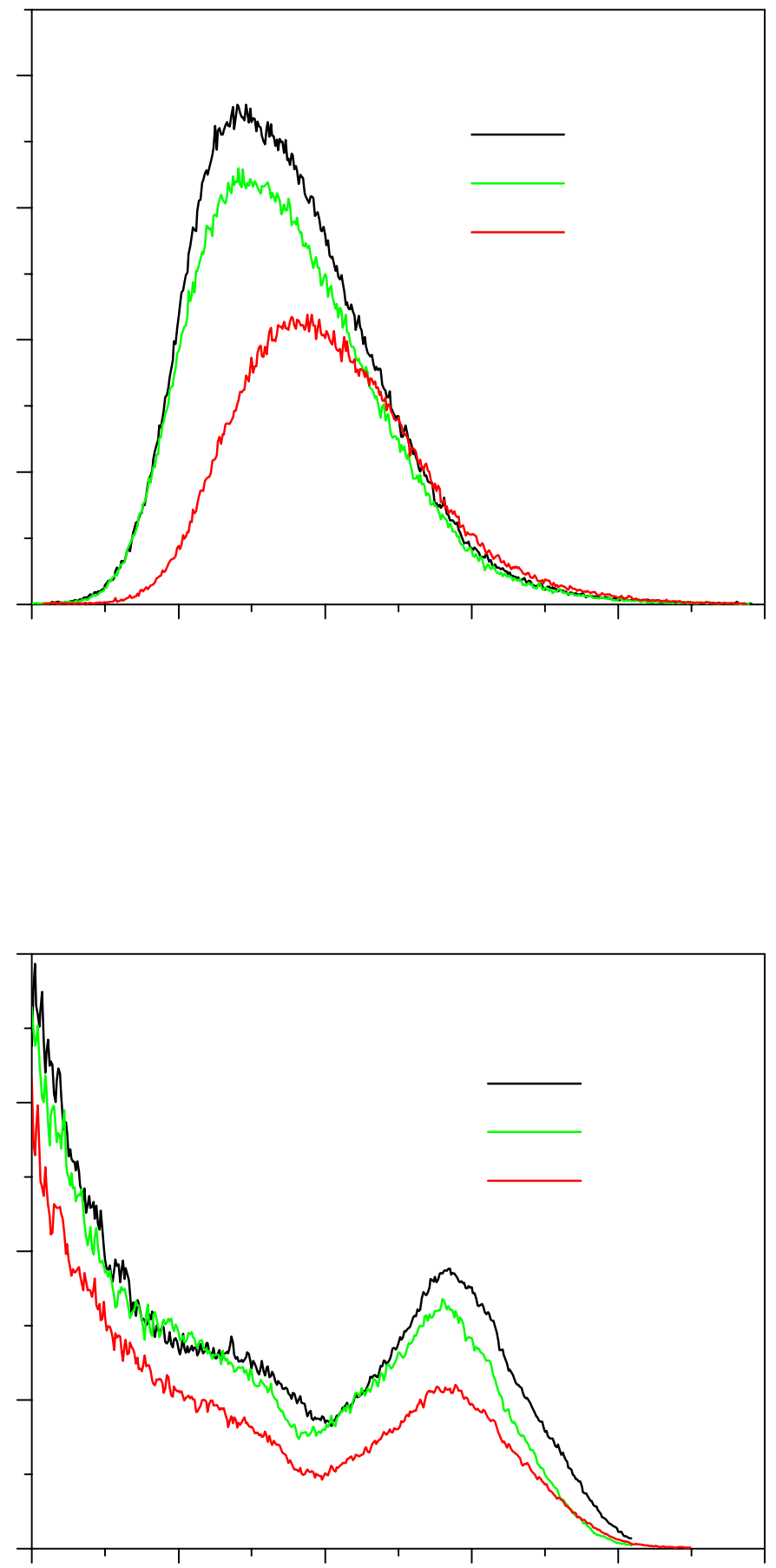

Figure 10. Fluorescence emission spectra (up) and excitation spectra (down) of $\mathbf{4 j} \mathbf{j}$ in different solvents 

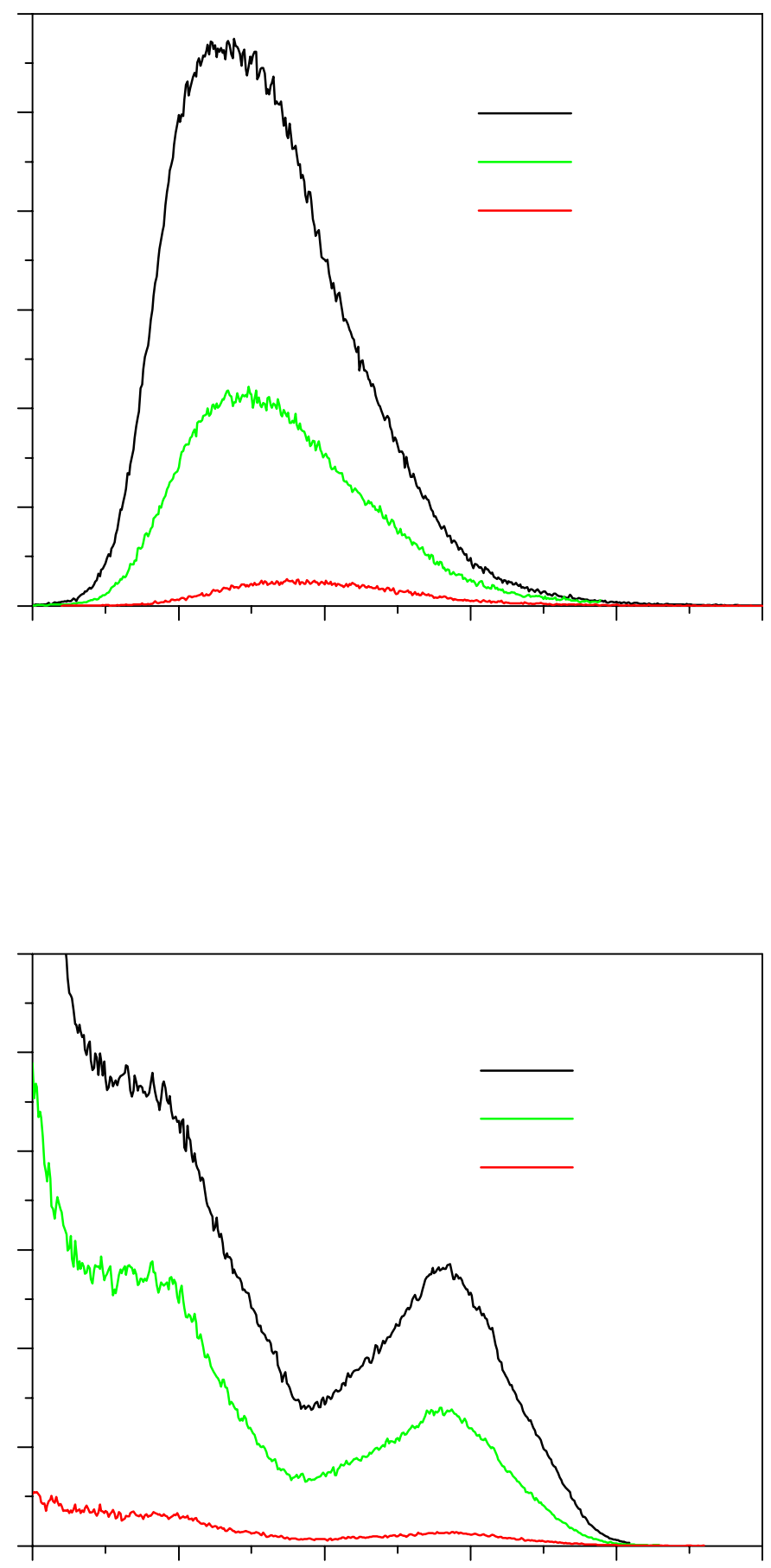

Figure 11. Fluorescence emission spectra (up) and excitation spectra (down) of $\mathbf{4 k}$ in different solvents 

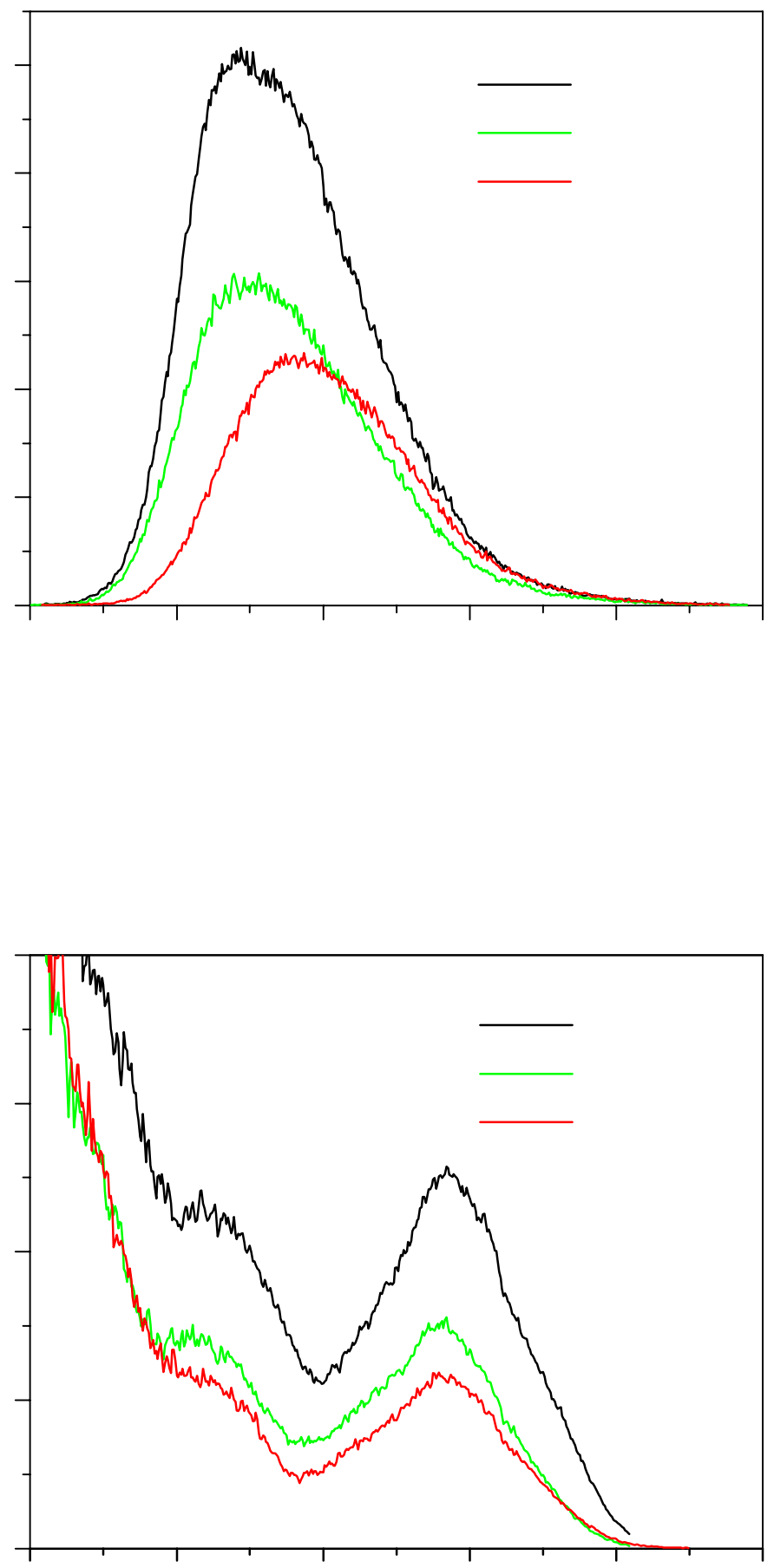

Figure 12. Fluorescence emission spectra (up) and excitation spectra (down) of $\mathbf{4 1}$ in different solvents 

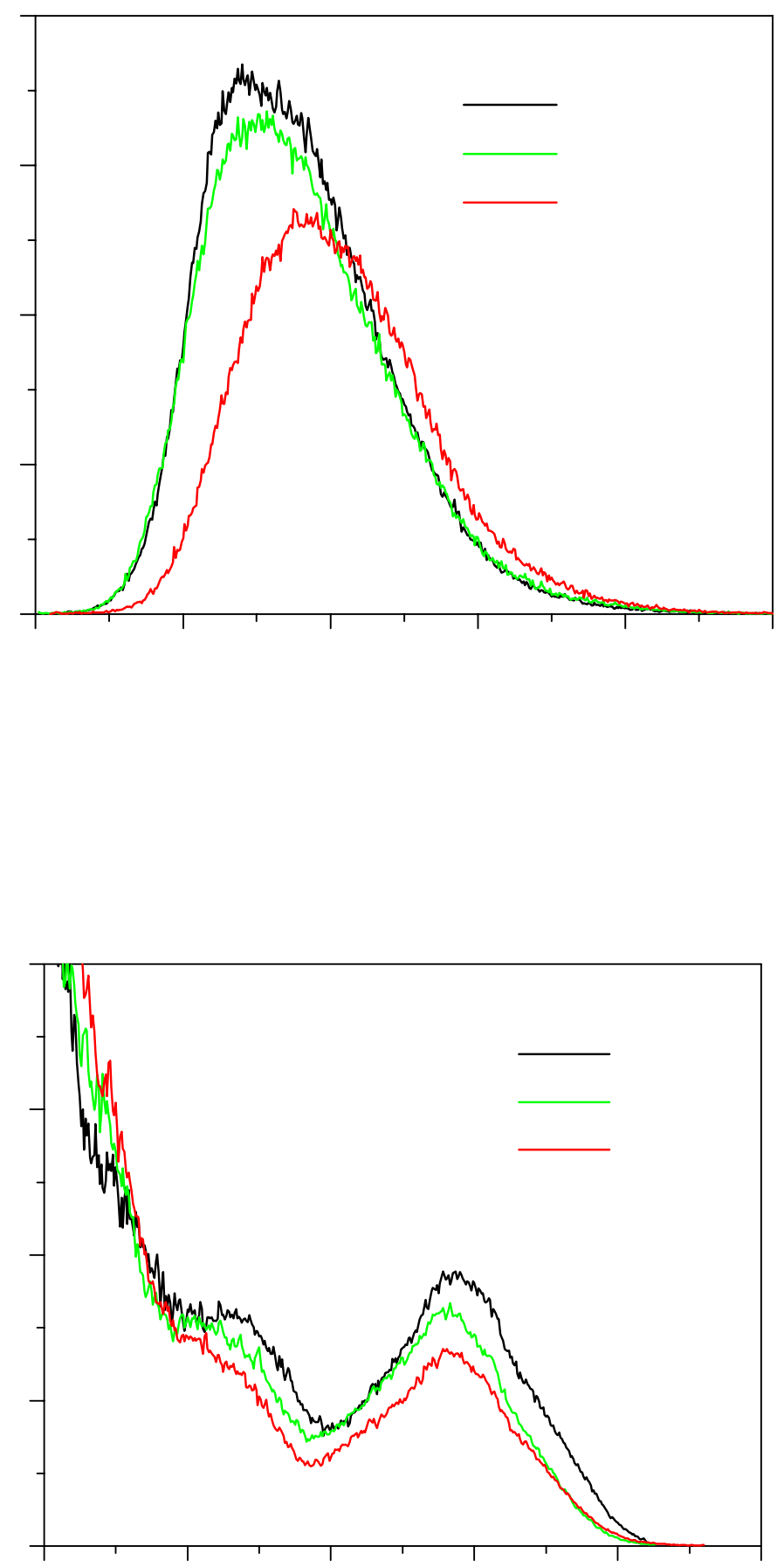

Figure 13. Fluorescence emission spectra (up) and excitation spectra (down) of $\mathbf{4 m}$ in different solvents 

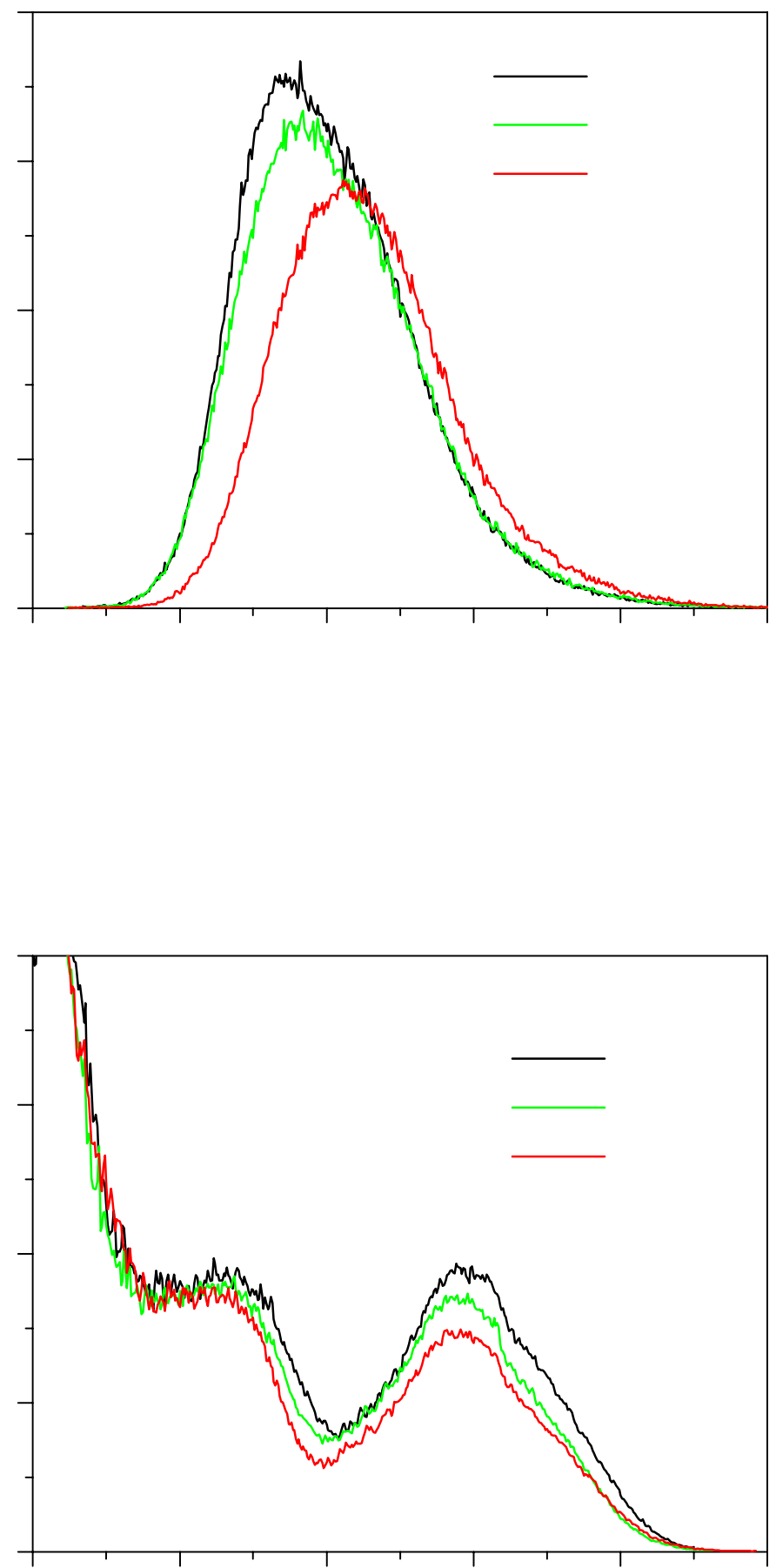

Figure 14. Fluorescence emission spectra (up) and excitation spectra (down) of $\mathbf{4 n}$ in different solvents 

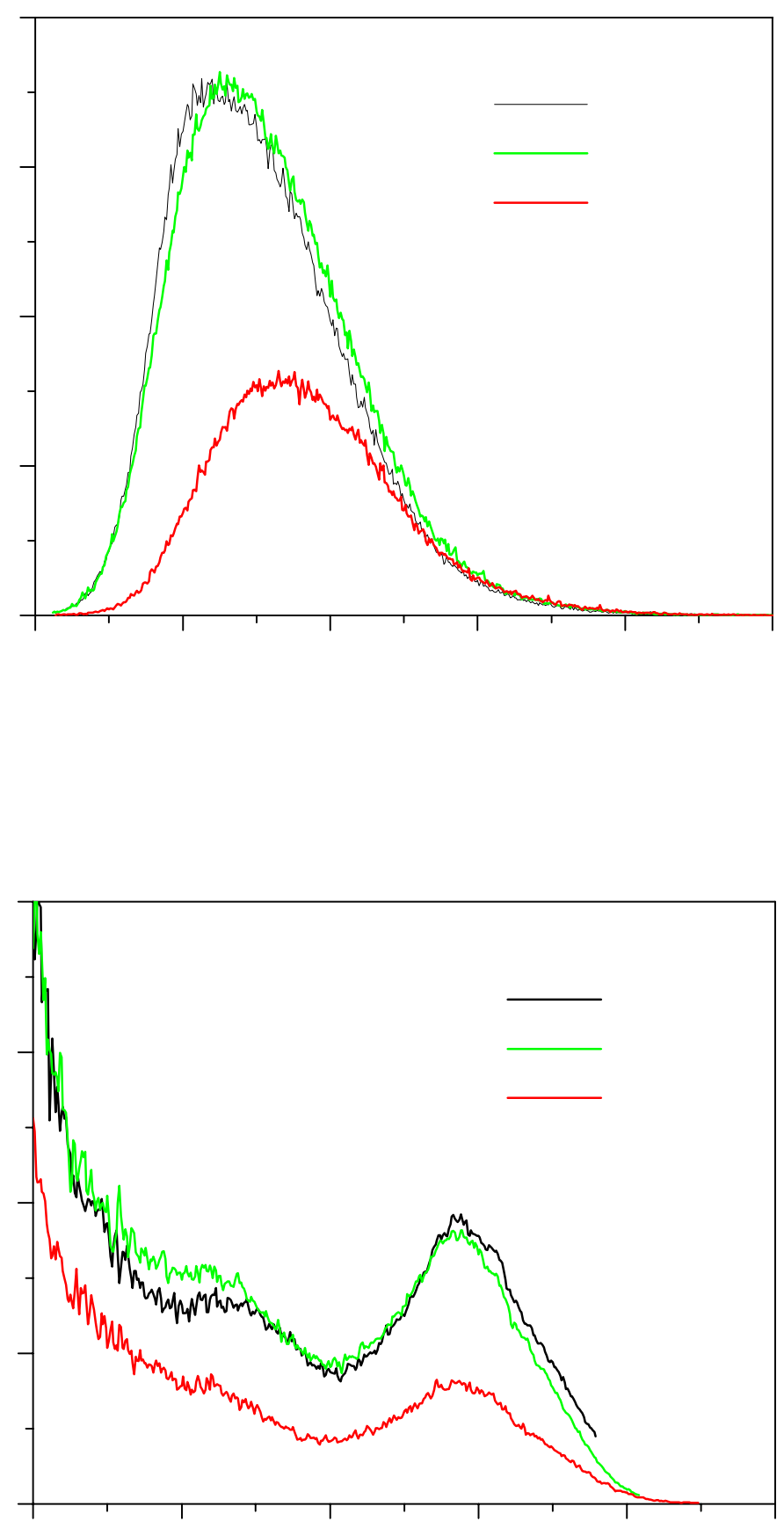

Figure 15. Fluorescence emission spectra (up) and excitation spectra (down) of $\mathbf{4 o}$ in different solvents 


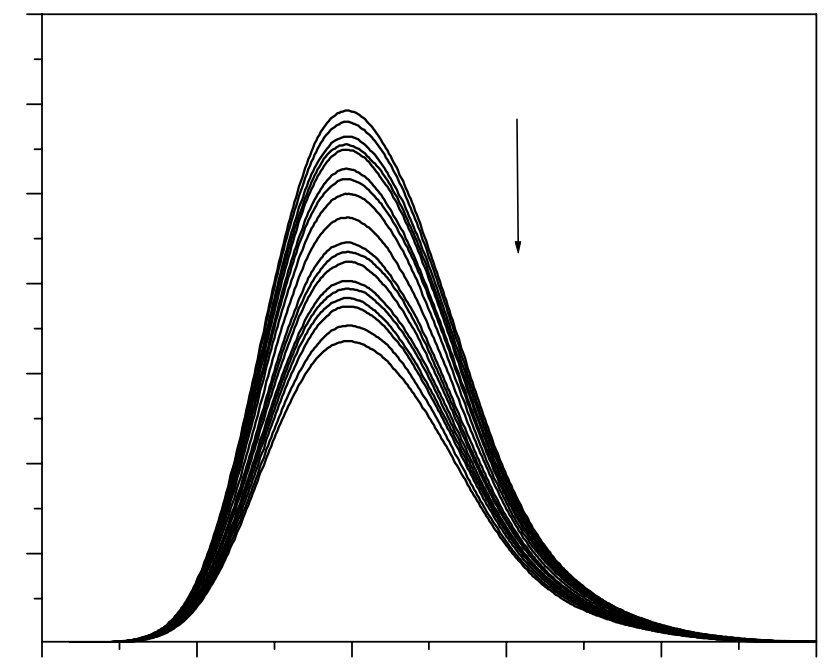

Figure 16. Spectrofluorimetric titration of $4 \mathbf{c}\left(\sim 10^{-5} \mathrm{M}\right.$ in $\left.\mathrm{MeOH}\right)$ with trifluoroacetic acid.

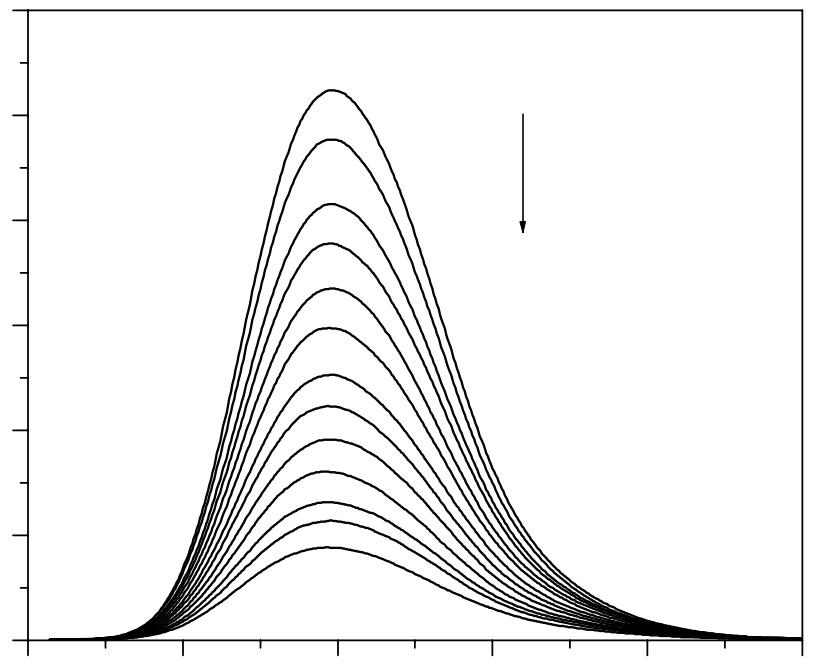

Figure 17. Spectrofluorimetric titration of $40\left(\sim 10^{-5} \mathrm{M}\right.$ in $\left.\mathrm{MeOH}\right)$ with methanolic triethylamine 
X-ray crystal structure analysis for 4 f: formula $\mathrm{C}_{17} \mathrm{H}_{12} \mathrm{~N}_{2} \mathrm{O}_{4} * \mathrm{H}_{2} \mathrm{O}, \quad M=326.30$, yellow crystal $0.35 \times 0.25 \times 0.20 \mathrm{~mm}, a=7.103(1), b=13.709(1), c=14.869(1) \AA ., V=$ $1447.9(2) \AA^{3}, \rho_{\text {calc }}=1.497 \mathrm{~g} \mathrm{~cm}^{-3}, \mu=0.940 \mathrm{~mm}^{-1}$, empirical absorption correction $(0.734$ $\leq T \leq 0.834$ ), $Z=4$, orthorhombic, space group $P 22_{1} 2_{1} 2_{1}$ (No. 19), $\lambda=1.54178 \AA, T=223$ $\mathrm{K}, \omega$ and $\varphi$ scans, 12370 reflections collected $( \pm h, \pm k, \pm l),[(\sin \theta) / \lambda]=0.60 \AA^{-1}, 2564$ independent $\left(R_{\text {int }}=0.033\right)$ and 2552 observed reflections $[I \geq 2 \sigma(I)], 231$ refined parameters, $R=0.027, w R^{2}=0.073$, max. residual electron density $0.12(-0.13)$ e $\AA^{-3}$, Flack parameter $-0.07(16)$, hydrogen atoms at water from difference fourier calculation, other calculated and refined as riding atoms.

Data set was collected with a Nonius KappaCCD diffractometer. Programs used: data collection COLLECT (Nonius B.V., 1998), data reduction Denzo-SMN (Otwinowski, Z.; Minor, W. Methods in Enzymology 1997, 276, 307-326), absorption correction Denzo (Otwinowski, Z.; Borek, D.; Majewski, W.; Minor, W. Acta Cryst. 2003, A59, 228-234), structure solution SHELXS-97 (Sheldrick,G.M. Acta Cryst. 1990, A46, 467-473), structure refinement SHELXL-97 (Sheldrick, G.M. Universität Göttingen, 1997), graphics SCHAKAL (Keller, E. 1997).

CCDC 610504 contain the supplementary crystallographic data for this paper. These data can be obtained free of charge at www.ccdc.cam.ac.uk/conts/retrieving.html [or from the Cambridge Crystallographic Data Centre, 12 Union Road, Cambridge CB2 1EZ, UK; fax: (internat.)+44(1223)336-033, E-mail: deposit@ccdc.cam.ac.uk].

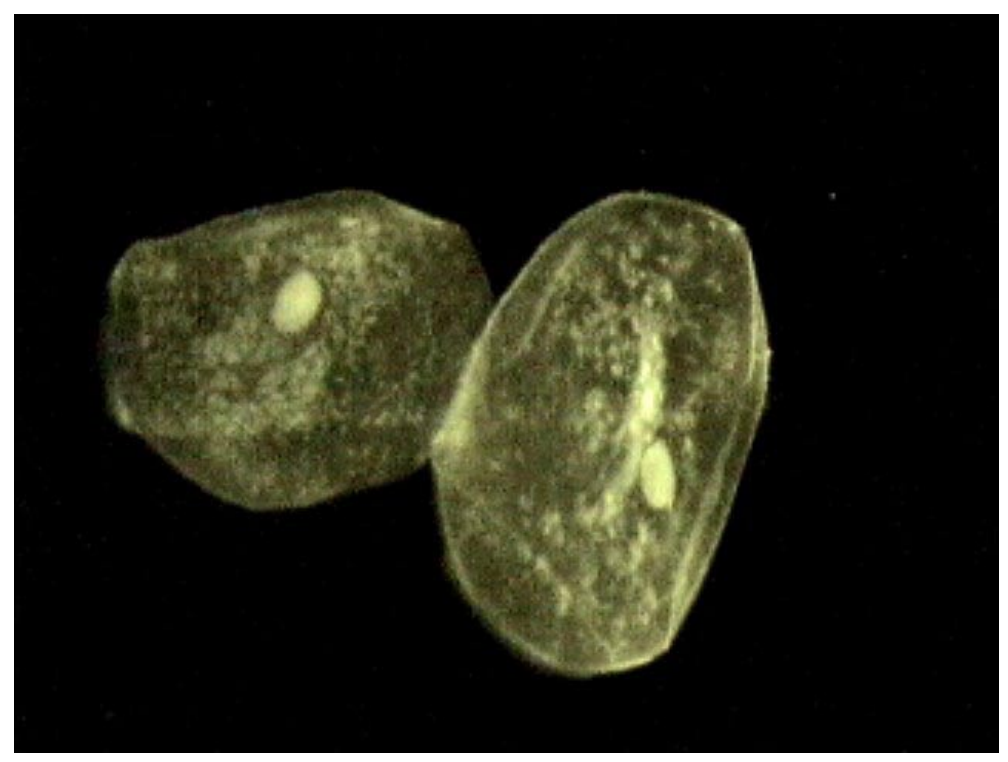

Figure 18. Image of cells stained by a solution of $\mathbf{4 a}$ in methanol/water $(1: 5 \mathrm{v} / \mathrm{v})$ under fluorescence microscope. 